\title{
Article
}

\section{The influence of a word's number of letters, spatial extent, and initial bigram characteristics on eye movement control during reading: Evidence from Arabic.}

Hermena, Ehab W., Liversedge, Simon Paul and Drieghe, Denis Available at https://clok.uclan.ac.uk/22520/

Hermena, Ehab W., Liversedge, Simon Paul orcid iconORCID: 0000-0002-85798546 and Drieghe, Denis (2017) The influence of a word's number of letters, spatial extent, and initial bigram characteristics on eye movement control during reading: Evidence from Arabic. Journal of Experimental Psychology: Learning, Memory, and Cognition, 43 (3). pp. 451-471. ISSN 0278-7393

It is advisable to refer to the publisher's version if you intend to cite from the work. http://dx.doi.org/10.1037/xIm0000319

For more information about UCLan's research in this area go to http://www.uclan.ac.uk/researchgroups/ and search for <name of research Group>.

For information about Research generally at UCLan please go to http://www.uclan.ac.uk/research/

All outputs in CLoK are protected by Intellectual Property Rights law, including Copyright law. Copyright, IPR and Moral Rights for the works on this site are retained by the individual authors and/or other copyright owners. Terms and conditions for use of this material are defined in the policies page. 
The Influence of a Words' Number of Letters, Spatial Extent, and Initial Bigram Characteristics on Eye Movement Control During Reading: Evidence from Arabic.

Ehab W. Hermena, Simon P. Liversedge, \& Denis Drieghe University of Southampton

Author Note

Ehab W. Hermena, Simon P. Liversedge and Denis Drieghe, Centre for Vision and Cognition, Psychology, University of Southampton, Southampton, England.

Correspondence regarding this article should be addressed to Ehab W. Hermena, Centre for Vision and Cognition, School of Psychology, Shackleton Building, University of Southampton, Highfield Campus, Southampton, SO17 1BJ, UK. Tel: +44(0)2380 595078, Email: ewh1g09@soton.ac.uk

This research was supported by a Leverhulme Trust Research Grant RPG-2013-205. 


\begin{abstract}
We conducted two eye movement experiments in which we used the typographical and linguistic properties of Arabic to disentangle the influences of words' number of letters and spatial extent on measures of fixation duration and saccade targeting (Experiment 1), and to investigate the influence of initial bigram characteristics on saccade targeting during reading (Experiment 2). In the first experiment, through the use of a proportional font, which is more natural-looking in Arabic compared to monospaced fonts, we manipulated the number of letters (5 versus 7$)$ and the spatial extent (wide versus narrow) of words embedded in frame sentences. The results obtained replicate and expand upon previous findings in other alphabetic languages that the number of letters influences fixation durations, whereas saccade targeting (as indicated by measures of fixation count and probability of skipping and re-fixation) is more influenced by the word's spatial extent. In the second experiment we compared saccade targeting measures (saccade amplitude and initial fixation location) in 6- and 7- letter words beginning with initial bigrams that were of extremely high frequency (ال the), relatively high frequency (لل to/for the), or beginning with the letters of the word stem. Our results showed negligible modulation of saccade targeting by initial bigram characteristics. The results also highlighted the importance of selecting the appropriate measures of initial fixation location (spatial versus character-based measures) during reading text rendered using proportional fonts.
\end{abstract}

Key words: Arabic reading, word length, saccade targeting 
During reading, readers make two types of decision regarding their eye movement behavior. The first of these decisions relates to when to terminate a fixation and move the eyes (fixation duration). The other decision relates to where to send the eyes next (e.g., measures of saccade amplitude, skipping, and initial fixation location). These decisions are mostly influenced by different properties of the text, that is, they are largely independent (Rayner \& McConkie, 1976; Rayner \& Pollatsek, 1981). Evidence suggests that fixation durations are mainly influenced by the demands of linguistic processing (e.g., word frequency and predictability), whereas saccade targeting measures are mostly influenced by low-level properties of the text such as inter-word spacing (see Rayner, 2009 for review). With a few exceptions (e.g., in Chinese: Bai, Yan, Liversedge, Zang, \& Rayner, 2008; Li \& Shen, 2013; and Arabic: Paterson, Almabruk, McGowan, White, \& Jordan, 2015), these aspects of eye movement control in reading of non-European languages remain understudied. Here we report experiments investigating the influence of Arabic words' number of letters, spatial extent, and characteristics of initial bigrams on eye movement control during reading.

\section{The Number of Constituent Letters and Spatial Extent of a Word}

In our first experiment we aimed to further separate and quantify the contributions of a word's number of letters and spatial extent on eye movement control during reading. Predominantly, the influence of the number of letters on eye movements has been referred to as word length effects. Indeed, one of the benchmark findings reported in the literature on eye movements in reading is the effect of word length. Numerous investigations have reported that compared to words with fewer letters, words that contain more letters are skipped less 
(Brysbaert, Drieghe, \& Vitu, 2005; Drieghe, 2008; Drieghe, Brysbaert, Desmet, \& De Baecke, 2004), attract longer fixation durations (e.g., Calvo \& Meseguer, 2002; Hyönä \& Olson, 1995; Juhasz, White, Liversedge, \& Rayner, 2008), and attract more fixations and re-fixations (e.g., Kliegl et al., 2004; Rayner \& McConkie, 1976; Rayner, Sereno, \& Raney, 1996).

Other investigations revealed that readers utilize the spaces between upcoming words, parafoveally, as a cue for word length (Inhoff, Radach, Eiter, \& Juhasz, 2003; Morris, Rayner, \& Pollatsek, 1990, Pollatsek \& Rayner, 1982; Rayner, Fischer, \& Pollatsek, 1998; Rayner \& Pollatsek, 1996; White, Rayner, \& Liversedge, 2005). Furthermore, these investigations reported that readers use word length information to program saccades to target the word center, the optimal viewing position, OVP (Nuthmann, Engbert, \& Kliegl, 2005; O’Regan \& LévySchoen, 1983; Vitu, O’Regan, \& Mittau, 1990). Typically, however, readers' initial fixation falls short of its target and lands between word beginning and center, at the so-called preferred viewing location (PVL, Rayner 1979; see also McConkie, Kerr, Reddix, \& Zola, 1988; McConkie, Kerr, Reddix, Zola, \& Jacobs, 1989; Rayner et al., 1998; Vitu et al., 1990). Evidence also showed that initial fixation locations are typically closer to word center in short words, relative to longer words (e.g., Bertram \& Hyönä, 2003). The location of the initial fixation on words has also been found to vary as a function of the launch site (or the location from which the saccade was launched into the target), with closer launch sites resulting in saccades landing further into the word (see McConkie et al., 1988).

A key feature common to almost all the above-cited investigations, is the use of monospaced fonts. In monospaced fonts letters occupy an identical amount of horizontal space, regardless of their visual complexity (e.g., the letters $i$ and $w$ in Courier New font). Thus, in these investigations the number of letters in a word and the amount of physical space the word 
occupies, or its spatial extent, were perfectly confounded (Hautala et al., 2011; McDonald, 2006).

McDonald (2006) used monospaced Monaco font in an experiment in which he presented readers with sentences containing 6- or 8-letter target words. The target words, and the remaining words in the sentences (2- to 10-letters), were rendered such that they all subtended the same visual angle through a font manipulation (horizontal scaling). For the target words, McDonald reported that the number of fixations, as well as fixation durations, increased significantly for 8- compared to 6-letter words. Word skipping, launch sites, and initial fixation locations, on the other hand, did not differ between the 6- and 8-letter conditions. However, for non-target words an inconsistent pattern emerged whereby initial fixation locations (measured in characters) shifted towards word centers as the number of letters increased. Thus, no clear conclusion could be made as to whether the number of letters of a word affects initial fixation location. Furthermore, the font manipulation used by McDonald (2006) was criticized for making the sentences look unnatural, and for increasing visual crowding in words with more letters (see Hautala et al., 2011).

To address these shortcomings, Hautala et al. (2011) compared eye movement measures on Finnish sentences written in both monospaced font (Courier New) in which 6-letter words subtended a larger spatial extent than 4-letter words, and proportional font (Arial), a key feature of which is that letter sizes vary naturally. Making use of the natural variability of letter sizes in Arial, Hautala et al. selected 4- and 6-letter words that subtended an identical spatial extent, also ensuring that these words were of equal spatial extent to the 4-letter words in the monospaced font. Hautala et al. also compared readers' eye movements on other word pairs from the stimuli sentences rendered in proportional font. These word pairs contained the same number of letters 
while extending over either a narrow or a wide spatial extent (a difference of about 7 pixels). Hautala et al.'s results were unequivocal. Saccade targeting measures (e.g., skipping probability, or the probability that word is not fixated, rather, the following fixation falls on the subsequent word; and saccade amplitudes, or the length of a saccade, reported in pixels) were influenced mainly by the spatial extent of the words, regardless of a word's number of letters. In contrast, fixation durations were clearly influenced by number of letters, regardless of spatial extent. With the findings of McDonald (2006) and Hautala et al. (2011) in mind, we aimed to further investigate the influence of the number of letters of a word, and its spatial extent, on eye movement control in our first experiment. We elected to investigate these effects during reading Arabic given the typographical characteristics of Arabic text. Chief amongst these is the fact that Arabic is rarely printed using monospaced fonts. Selecting a commonly available Arabic proportional font allowed us to present readers with 5- or 7-letters target words that were of identical spatial extent that were either narrow or wide (average difference was about 10 pixels), with no font manipulations. Using such stimuli allowed us to expand upon the valuable findings reported by Hautala et al. (2011), while avoiding the caveats they highlighted for comparing eye movement measures across two different fonts (e.g., how visuo-spatial properties of the fonts, like variability in letter spacing, may influence eye movement behavior, see also Rayner, Slattery, \& Bélanger, 2010).

Our investigation also aimed to extend the findings reported by Paterson et al. (2015) who were the first to examine word length effects in reading Arabic. Paterson et al. used a proportional font for their stimuli, however, they selected stimuli, similar to monospaced fonts, that featured a very strong positive correlation between number of letters and spatial extent $(r=$ .93). As such, whilst very informative, Paterson et al.'s experiment was unable to disentangle 
effects of number of letters and spatial extent. In their experiment, Paterson et al. presented native Arabic readers with 3-, 5-, and 7-letter words. The authors reported increased skipping probabilities for shorter words relative to longer words, and longer saccades into longer words. Paterson et al. also reported that initial fixation locations were closer to word center for shorter words relative to longer words. Additionally, fixation durations increased for longer words. Our investigation aimed to replicate these findings and distinguish between the effects of the number of letters of a word and a word's spatial extent in Arabic through orthogonally manipulating these two factors.

Previous investigations have, however, highlighted that, in addition to the word's spatial extent, saccade targeting into a word can be influenced by other linguistic variables (e.g., Hyönä, 1993; 1995; White \& Liversedge, 2004; 2006). As will be detailed below, these investigations revealed that the saccade targeting system is somewhat sensitive to the characteristics of the letter string at a word beginning. In our second experiment we aimed to further investigate, and expand upon these findings concerning the influence of the characteristics of the initial letter string of a word on the saccade targeting system.

\section{Saccadic Targeting: Initial Bigram Characteristics}

As mentioned above, in addition to the influence of a word's spatial extent, a number of investigations have shown that some linguistic variables may also modulate saccade targeting. One such linguistic variable is word predictability which was found to influence skipping probabilities (more skipping of predictable words), but not the initial fixation location within a word (Rayner, Binder, Ashby, \& Pollatsek, 2001; see also Ehrlich \& Rayner, 1981; Rayner \& 
Well, 1996; Vainio, Hyönä, \& Pajunen, 2009). This shows that a distinction needed to be made between the factors that influence the decision of which word to target, and the factors influencing where a saccade lands within a word. The latter decision seems to be influenced less by linguistic processing of the upcoming word. Another linguistic variable that has been examined in relation to saccadic targeting is morphological complexity. Yan et al. (2014) reported that in Uighur readers landed closer to a word beginning in words that had more suffixes compared to words with fewer suffixes (suffix numbers ranged between 0 and 3 , and the maximum amount of initial fixation location shift was 0.6 character). This study thus indicated some influence of the linguistic processing of an upcoming word on the programming of a saccade into that word. Notably, Uighur is written using Arabic letters, and also like Arabic, it is read from right to left. However, unlike Arabic, Uighur features highly concatenative morphology, whereby numerous suffixes can be added to a word stem to modify its meaning (see Abu-Rabia, 2007; Frost, Kugler, Deutsch, \& Forster, 2005 for more on Semitic morphology).

Of particular interest for the current study are the findings that the linguistic factors of orthographic regularity and familiarity (both operationalized as frequency) of a word's initial letter sequence were also found to modulate saccade targeting in single word tasks (making saccades to a target word from a fixation cross, e.g., Beauvillain \& Doré, 1998; Beauvillain, Doré, \& Baudouin, 1996; Doré \& Beauvillain, 1997), and in reading (e.g., Hyönä, 1995; Plummer \& Rayner, 2012; Radach, Inhoff, \& Heller, 2004; Vonk, Radach, \& van Rijn, 2000; White \& Liversedge, 2004; 2006).

To explain this effect, Hyönä (1993) put forth an attraction hypothesis whereby less frequent letter strings would 'pop out' in the parafovea and attract fixations. Hyönä (1995) subsequently reported that Finnish words that contained highly irregular (very low frequency) 
initial letter strings attracted initial fixations that were about 0.5 character closer to word beginning compared to words that began with regular letter sequences. Similarly, White and Liversedge (2004) reported that initial fixation location shifted towards word beginning (0.3 0.5 characters) in words beginning with misspelled bigrams. Similar findings were also reported by Plummer and Rayner (2012) for words beginning with misspelled trigrams (but see Liu, Li, Han, \& Li, 2014 who did not find a similar effect in Chinese). Additionally, White and Liversedge (2006) reported that, for correctly spelled words, initial fixation location shifted towards word beginning in words starting with irregular letter sequences, compared to words starting with more regular sequences. However, it is important to note that all the studies in which an influence of linguistic properties on saccade targeting (mainly initial fixation location) was observed, the effect size obtained was quite modest.

In Experiment 2 we used Arabic as a medium to investigate the influence of initial bigram characteristics on saccade targeting. We chose Arabic since it features a bigram ال or the that is of extremely high frequency as an initial bigram. Our aim was to examine whether the presence of such an extremely frequent initial bigram would result in readers making saccades further into words than would be the case in the absence of the bigram. Such findings would complement previous findings in which very low frequency initial letter clusters resulted in initial fixations deviating modestly towards word beginnings.

\section{Experiment 1}

Using a commonly available proportional font, we selected target words that were either 5- or 7-letters long and subtended identical spatial extents that were either narrow or wide (see 
Figure 1 Panel A). Thus, we were able to decouple the linear relationship between number of letters and spatial extent observed in monospaced fonts.

Our first hypothesis was that, similar to Hautala et al. (2011), saccade targeting measures would be influenced by a target word's spatial extent, not number of letters. Essentially, narrow extent words would be skipped more, receive shorter saccades, and have initial fixations that land closer to word center, compared to words with a wider extent.

With regards to initial fixation location, we report two different measures of initial fixation location on the target word. The first of these measures is initial fixation location measured in letters (initial fixation letter), starting with the space before the target word. The second measure is initial fixation location as a percentage of the spatial extent of the interest area that contains the target (starting from the right boundary of this interest area, and including the space before the word, recall that Arabic is read right to left). Thus, whereas the first measure is sensitive to the linguistic unit of the letter, the latter measure is more sensitive to the spatial extent of the word (see Figure 1 Panel C). This enabled us to compare our initial fixation location findings with those of Paterson et al. (2015). Recall that in their investigation, despite using a proportional font, the linear relationship between the target words' spatial extent and number of letters was largely preserved, as in previous investigations using monospaced fonts. Paterson et al. reported that initial fixation location measures yielded similar patterns when initial fixation location was measured by dividing the words into: (i) unequal spaces that corresponded exactly to letter location and extent, and (ii) equal spaces based on word's spatial extent divided by number of letters, such that each space did not necessarily correspond to the exact letter locations and extent. 
$<$ Insert Figure 1 about here $>$

Our second hypothesis was also informed by previous findings whereby fixation duration measures were influenced by number of letters, and not by spatial extent (e.g., Hautala et al., 2011). We thus expected that 7-letter words would attract longer fixation durations than 5-letter words. Additionally, and given that the spatial extent of the 5- and 7-letter words was either narrow or wide, we would be able to examine any possible influence on fixation durations of increased visual crowding in the narrow condition.

Finally, previous findings provided mixed accounts regarding what influences the probability of refixation. Some findings showed that words containing more letters attract more refixations (e.g., McDonald, 2006); while other findings showed that increased spatial extent is responsible for this effect (e.g., Hautala et al., 2011). The latter pattern would be more consistent with the suggestion that readers refixate a long word in order to bring the remainder of it into foveal vision (e.g., Vergilino-Perez, Collins, \& Doré-Mazars, 2004). We hypothesized that this is the more likely scenario. However, McDonald's findings that increased probability of refixation accompanied increases in number of letters may have resulted from the fact that words containing more letters in his study suffered more visual crowding. Thus, if we find evidence for increased refixation rates in narrow extent words, this may indicate that refixations may be necessary, in addition to increased fixation durations, when processing words with visually crowded letters. 


\section{Participants}

Thirty-six adult native Arabic speakers were paid $£ 15$ for participation in the eye tracking procedure. To be explicit, only participants who were born in Arabic speaking countries, with Arabic as their first language, were classed as native readers of Arabic and were allowed to participate. All participants were UK residents or visitors. The participants ( 23 females) had mean age of 32.5 years $(\mathrm{SD}=8.7$, range $=18-47)$. All participants had normal or corrected vision, and all reported being able to clearly see the words on the screen during a practice block. The majority of participants spoke and read English as a second language. All participants read Arabic text regularly (daily or weekly). The participants were naïve as to the exact purpose of the experiments.

For the stimuli norming tasks detailed below, we used participants from Amazon Mechanical Turkers (AMTs). These participants were thus not from the same population sample who took part in the eye tracking procedure. The exact number of AMTs participating in each task is detailed below. AMTs were paid $£ 10-15$, depending on the number of tasks in which they participated. All AMTs were adult skilled Arabic readers, otherwise they would not have been able to complete the numerous 'quality check' tasks we embedded in our norming procedures (e.g., providing accurate definitions of the target words in complete and grammatically sound sentences; and placing the target words in original sentences that are grammatically sound). All tasks were time capped and so only skilled readers were able to complete the work in the time allowed. Any AMTs' responses that did not meet our strict criteria were excluded from our analyses and the tasks were 're-published' for new AMTs. In other words, data from AMTs 
whose work did not pass our quality checks was not included in the norming, and additional AMTs were recruited to replace them.

\section{Reading skill screening for participants in the eye tracking procedure.}

The participants in the eye tracking procedure were furthermore screened for proficiency of reading Arabic and accuracy of phonological decoding. To this end, they were required to perform a text reading aloud task (346 words), while being audio-recorded, prior to eye tracking. And subsequent to the eye tracking procedure, the participants were required to read aloud, also while being audio recorded, a list of single words (36 words carrying Arabic diacritical marks which add vowel sounds to the letters). Accuracy in decoding diacritical marks was relevant to another investigation of reading in Arabic which we ran simultaneously, however this allowed us to further examine the linguistic proficiency of the participants taking part in the current study.

All 36 participants were highly accurate in text reading (mean percentage of words read accurately $=97.3 \%, \mathrm{SD}=0.98$, range $=95.6-100 \%$ ). Finally, for the single word reading aloud, all 36 participants were highly accurate (mean word reading accuracy $=93.5 \%, \mathrm{SD}=7.3$, range $=84.2-100 \%)$.

\section{Stimuli}

Thirty-seven sets of four target words (total 148 words) were created ${ }^{1}$. The target words were embedded in frame sentences in one of four conditions: 5 letters, narrow extent (5-Narrow, for short); 5 letters, wide extent (5-Wide); 7 letters, narrow extent (7-Narrow); and 7 letters, wide 
extent (7-Wide). With the exception of the target word, the four frame sentences were identical in 23 of the stimuli sets (see Figure 1 Panel A). For the remaining sets the frame sentences were identical only until the target word.

We chose our targets from two of the largest letter bins in the Aralex corpus (Boudelaa \& Marslen-Wilson, 2010), the 5- and 7-letter word bins. We chose the font Traditional Arabic from a number of proportional fonts based on an extensive norming procedure (detailed in Appendix 1).

\section{Stimuli matching and norming.}

To begin with, in order to match the target 5- and 7-letter words on spatial extent, we rendered the words into image files and measured the spatial extent of each word in pixels. On average, wide words were 10 pixels wider than narrow words, and this difference was statistically significant $(t(158)=37.1, p<.001$, see Table 1$)$.

\footnotetext{
$<$ Insert Table 1 about here $>$
}

Subsequently, target words in the four experimental conditions (5-Narrow, 5-Wide, 7Narrow, 7-Wide, see Table 1 for full descriptives) were matched on orthographic frequency using the Aralex corpus (Boudelaa \& Marlsen-Wilson, 2010). Log-transformed word frequency did not differ between the conditions $(F<1)$. Additionally, using a 5-point scale $(5=$ word is very common; 1 = word is rare), there was no difference between the conditions on subjective ratings of target word commonness (10 Amazon Mechanical Turkers' ratings per word, $F<1$ ). 
We also checked if the visual density of the target words differed between the conditions. We used the percentage of dark pixels in the interest area containing the target word as a proxy of visual density. Overall, there was a significant difference between the conditions for visual density $F(3,156)=7.87, p<.001$. Post hoc Tukey HSD tests revealed that only the difference between the 5 -wide and 7 -wide conditions was significant $(p<.001)$ but the small difference between these two conditions (about 5\% more dark pixels in 7 -wide words, see Table 1 ) is relatively negligible. None of the other contrasts were significant (all $p \mathrm{~s}>.12$ ). Related to the same point, we checked whether the target words in any of the conditions contained more narrow letters (the three letters I, J, e, which also happen to be visually simpler than the remaining, wider, 25 letters of the Arabic alphabet letters, e.g., س/s/s/sh/, ش/h/, etc.). As Table 1 shows, there was no difference between the conditions in the amount of wide letters included in the target words $(F<1)$.

The Traditional Arabic font we used allows letters to overlap in vertical space, (see Figure 1 Panel B), such that one letter can occupy the space above the following letter. This type of Arabic script is named Ruq'a, and can be contrasted to scripts which do not allow vertical letter overlaps known as Naskh scripts (Wightwick \& Gaafar, 2005). Both types of scripts are very common in Arabic, and are also taught as handwriting styles (Wightwick \& Gaafar, 2005). We checked whether the amount of vertical letter overlaps differed between the conditions. There was a significant difference between the conditions $(F(3,156)=31.8, p<.001$, see also Table 1). Post hoc Tukey HSD tests showed that words in the 7-Narrow condition contained more letter overlap compared to 5-Narrow words and 7-Wide words $(p \mathrm{~s}<.001)$. In addition, 7Wide words also contained more letter overlaps than 5-Wide words $(p<.001)$. However, there was no difference between 5-Wide and 5-Narrow words $(p>.10)$. Thus, for the target words we 
used, increased letter vertical overlap, rather than increased inclusion of narrow letters or increasing visual density, allowed for the inclusion of 7-letter words that subtend the same spatial extent as 5-letter words. Recall that this is a natural feature of Arabic Ruq'a scripts, not an experimental manipulation. Note also that obtaining target words in which different numbers of letters subtend the same spatial extent would be possible using the other Arabic proportional fonts that do not allow for vertical letter overlap (i.e., in Naskh scripts), given the natural variability in letter sizes in proportional fonts (see e.g., Hautala et al., 2011).

In each of the stimuli sets, the target words belonged to the same syntactic category. The target words were also matched on morphological complexity (i.e., the presence or absence of prefixes and suffixes, as well as relative complexity of root-form morpheme structures).

We obtained 10 cloze predictability ratings for the target word within each sentence. In this procedure, 10 participants were given sentences up to, but not including, the target word, and were asked to complete the sentence. With the exception of 14 of the target words produced by the Amazon Mechanical Turkers (AMTs) raters, none of the other target words were predictable (produced on zero occasions by the raters). After changing the pre-target context of the sentences containing these 14 words, we re-normed the new sentences by 10 additional raters and this revealed that these 14 words were no longer predictable (produced on zero occasions by the raters).

Finally, we obtained ratings of sentence structure naturalness for all target sentences on a 5-point scale ( 1 = structure is highly unusual, 5 = structure is highly natural). 10 ratings per sentence were obtained from 10 AMTs raters, and these indicated that sentence structure for all stimuli in all conditions was highly natural (Table 1), with no difference between the conditions $(F<1)$. 
Eye tracking data for the two experiments reported here were collected in one testing session, with the stimuli from both experiments acting as filler items for each other. Thus, in addition to the stimuli for Experiment 1, the participants were presented with: 45 stimuli from Experiment 2, additional 58 filler items, and 11 practice sentences. Thus each participant read 150 sentences in total.

All sentences were written and displayed on a single line and in natural cursive script. The text was rendered in Traditional Arabic font, size 18 (roughly equivalent to English text in Times New Roman font size 14).

\section{Apparatus}

An SR Research Eyelink 1000 tracker was used to record participants' eye movements during reading. Viewing was binocular, but eye movements were recorded from the right eye. The eye tracker sampling rate was set at $1000 \mathrm{~Hz}$. The eye tracker was interfaced with a Dell Precision 390 computer, with a 20 inch ViewSonic Professional Series $P 227 f$ CRT monitor. Monitor resolution was set at $1024 \times 768$ pixels. The participants leaned on a headrest to reduce head movements. The words were in black on a light grey background. The display was $81 \mathrm{~cm}$ from the participants, and at this distance, on average, 3.2 characters equaled $1^{\circ}$ of visual angle.

The participants used a VPixx RESPONSEPixx VP-BB-1 button box to enter their responses to comprehension questions and to terminate trials after reading the sentences.

When participants read reading skill screening materials aloud their voices were recorded using a standard digital voice recorder. 


\section{Design}

The number of letters and spatial extent of the target words were the two within-subjects independent variables. Sentences in the experimental conditions were counterbalanced using a Latin square, and presented in random order. Thus, participants saw only one sentence out of each set, and an equal number of target stimuli from all conditions.

\section{Procedure}

This experiment was approved by the University of Southampton Ethics Committee. Upon arrival at the lab, participants were given instructions for the experiment. Consenting participants subsequently read aloud the reading skill screening text. This was followed by the eye tracking procedure. Finally, participants' accuracy in phonological decoding ability was assessed as explained above.

The eye tracker was calibrated using a horizontal 3-point calibration at the beginning of the experiment, and the calibration was validated. Calibration accuracy was always $<0.25^{\circ}$, otherwise calibration and validation were repeated. Prior to the onset of the target sentence, a circular fixation target $\left(\right.$ diameter $\left.=1^{\circ}\right)$ appeared on the screen in the location of the first character of the sentence. If a stable fixation was detected on the target (i.e., the eye tracker detecting that the eye remained on the fixation target for $50 \mathrm{~ms}$ ), the display changed and the sentence appeared, otherwise recalibration and validation were performed.

The participants were told to read silently, and that they would periodically be required to use the button box to provide a yes/no answer to the questions that followed some sentences. 
Participants were allowed to take breaks, following which the tracker was re-calibrated. The testing session, including the reading skill screening tasks, the eye tracking procedure, and breaks lasted 60-80 minutes, depending on how many breaks a participant took.

\section{Results}

For all reported analyses, fixations with durations shorter than $80 \mathrm{~ms}$, or longer than 800 ms were removed. However, fixations shorter than $80 \mathrm{~ms}$ that were located within 10 pixels or less $\left(0.31^{\circ}\right.$ of visual angle approximately) from another longer fixation, were merged into the longer fixation. Along with removing trials in which blinks occurred, this resulted in removing approximately $3 \%$ of all data points. The data cleaning affected all experimental conditions equally (mean number of observations per condition $=323, \mathrm{SD}=5.5$, range $=315-326$ ). Furthermore, for each of the fixation duration measures, we removed data points \pm 2.5 standard deviations away from the mean fixation duration per participant and condition as outliers.

Comprehension questions followed $30 \%$ of all target sentences in the eye tracking procedure. To these, participants responded accurately on average $90 \%$ of the time $(\mathrm{SD}=5.3$, range $=82-100 \%$ ). There were no differences between the accuracy scores across the conditions $(F<1)$.

In addition to fixation duration and saccade targeting measures for the target words, we also report launch site given its modulating influence on initial fixation location (Hyönä, 1995; McConkie, Kerr, \& Dyre, 1994; McConkie et al., 1988; Radach \& McConkie, 1998; White \& Liversedge, 2004). We also report first pass refixation probability (or the probability that the 
word receives another fixation during first pass processing), and total fixation counts (or the total number of fixations the word received in first pass and subsequent processing).

As discussed above, we report initial fixation location measured in letters, and also as a percentage of the extent of the interest area containing the target word. To calculate initial fixation letter, similar to Paterson et al. (2015), we split the interest area into 6 or 8 regions, for the 5- and 7-letter words, respectively, each region containing one letter, plus one additional region for the space before the target word. These regions subtended different spatial extents reflecting the natural variation in character size in Arabic script. When characters were rendered such that they occupied the same vertical space (see Figure 1 Panel B), the region containing these characters was coded as the sum of numbers of the two letter positions divided by 2 (e.g., if letters 2 and 3 overlapped, the region containing these letters was coded as 2.5$)^{2}$.

We used the lme4 package (version 1.1-12, Bates, Maechler, \& Bolker, 2011) within the R environment for statistical computing (R-Core Development Team, 2013) to run linear mixed models (LMMs). The fixed factors of all models were the experimentally manipulated conditions of number of letters and spatial extents of target words and their interaction. Subjects and items were treated as random variables. We began our analyses with the 'full' random structure for the models (e.g., Barr, Levy, Scheepers, \& Tily, 2013) that included slopes for the main effects and their interactions. The random structure was systematically trimmed when failure to converge occurred, first by removing correlations between random effects, and if necessary also by removing their interactions. For each contrast we report beta values $(b)$, standard error $(S E)$, and $|t|$ or $|z|$ statistics. We performed log transformation of fixation durations' data to reduce distribution skewing (Baayen, Davidson, $\&$ Bates, 2008). Successive differences contrasts were run in which the intercept corresponds to the grand mean. For all analyses reported, Table 2 
contains the descriptive statistics, Table 3 contains the outputs of the LMMs for the saccade targeting measures, and Table 4 contains the output of the LMMs for fixation durations, fixation counts and refixation probabilities. For the measures of skipping (Table 3) and first pass refixation probability we used logistic generalized linear mixed models (GLMMs).

$<$ Insert Tables $2-4$ about here $>$

\section{Launch Site}

As Table 2 illustrates, average launch site is almost identical for all the conditions, with no significant differences meaning that any differences in initial fixation location are not a result of launch site differences.

\section{Saccade Targeting Measures}

Skipping Probability. Skipping probability was strongly influenced by the spatial extent of the target words such that wide words were skipped significantly less than narrow words. There was no effect of number of letters and no interaction.

Saccade Amplitude. Readers made longer saccades into wide words, relative to narrow words. There was no effect of the number of letters and no interaction.

Initial Fixation Letter. Readers landed about 0.5 character further in 7-letter compared to 5-letter target words (see Table 2), regardless of the spatial extent of the targets. This difference was statistically significant (Table 3 ). 
Initial Fixation Location as Percentage of Interest Area Extent. Readers landed about $2.7 \%$ further into the narrow words, compared to wide words, regardless of the number of letters. This difference was statistically significant (see Tables $2 \& 3$ ).

\section{Fixation Duration Measures}

Removing outliers resulted in removing $0.9 \%, 3.1 \%$, and $0.6 \%$ of data points for first fixation (the duration of the first fixation on the target word), single fixation (the duration of the first fixation on the target word when it received only one fixation), and gaze duration (the sum of all fixations on the target word during first pass reading) measures, respectively. In all three measures there were increases in fixation duration for 7- relative to 5-letter words, regardless of spatial extent (Table 3). These increases were statistically significant in single fixation and approached significance in gaze duration (Table 4). There was also a numerical trend in all three measures whereby fixation durations were increased for narrow relative to wide extent words.

\section{Other Processing Measures}

Wide extent words had significantly higher first pass refixation probability and had increased total fixation counts compared to narrow words. For both measures, there was no effect of number of letters and no interactions.

\section{Discussion}


In our experiment we were able to decouple the linear relationship between number of letters and spatial extent that is observed in investigations that used monospaced fonts to investigate word length effects. With regard to saccade targeting measures, our results show clearly that spatial extent, not number of letters, influenced the probability of word skipping thus replicating the findings reported by Hautala et al. (2011) who also controlled spatial extent and number of letters. Our results also replicate the findings from investigations using monospaced fonts in which the relationship between number of letters and spatial extent was linear, and longer words (of wider extent and containing more letters) were skipped less than shorter words (e.g., Brysbaert et al., 2005; Drieghe et al., 2004).

The results obtained in the saccade amplitude measure also reflect the influence of the target words' spatial extent, not the number of letters, and suggest that readers target word centers for landing: Wider words necessitate slightly longer saccades than narrow words so that fixations land at a landing site close to word center (see Hautala et al., 2011). Our results are also compatible with those of Paterson et al. (2015) who reported that readers made longer saccades into Arabic words with more letters, compared to words with fewer letters. Recall that Paterson et al. selected target words in which the number of letters correlated positively and strongly with spatial extent, thus, in effect, readers were making longer saccades into words with wider spatial extents.

With regard to initial fixation location, the effects obtained were not due to any variability in launch site. Our results suggested that number of letters influenced initial fixation location, when measured in letters. However, the results also suggested that the words' spatial extent exclusively influenced initial fixation location, when measured as a percentage of spatial extent 
of the interest area containing the target. These patterns are clearly at odds and require clarification.

We suggest that our results support the hypothesis that only spatial extent influences the saccade targeting measure of initial fixation location, not number of letters. As illustrated in Figure 1 Panel C, the obtained effect of number of letters on initial fixation location measured in letters was most likely due to the initial fixation pixel corresponding to a letter of a higher ordinal value in 7-compared to 5-letter words, given that there are more letters occupying the same spatial extent in the former condition. In other words, whereas the fixated pixel corresponds to the third letter in a 7-letter word, a fixation on the same location (pixel) would correspond to the second letter of a 5-letter word ${ }^{3}$. We suggest that this is more likely to be the reason for this effect, than actually an effect of number of letters on initial fixation location.

The discrepancy between our letter-based and spatial initial fixation location measures contrasts with Paterson et al.'s (2015) findings. Recall that Paterson et al.'s findings that initial fixation location results were similar when measured in character spaces and when measured in spaces that did not correspond to letter locations. Compared to Paterson et al., it is likely that the discrepancy between our two measures of initial fixation location was due to the decoupling of the linear relationship between the number of letters and spatial extent of our target words. We achieved this decoupling through presenting readers with target words that contained different number of letters, yet subtended identical spatial extent.

Indeed, initial fixation locations reported as percentage of interest area extent (Table 2) suggest that the readers aimed to land at the center of the target words (i.e., the optimal viewing position), but undershot and landed before the word center (i.e., the preferred viewing location, Rayner, 1979) in all conditions. Also, average initial fixation location was slightly closer to 
word beginning in wider extent words relative to narrow words, despite longer saccades into wider words. This is in line with classic findings based on monospaced fonts (e.g., McConkie et al. 1988; McConkie et al., 1989; Rayner, 1979; Rayner et al., 1996; Rayner et al., 1998; Vitu, O'Regan, Inhoff, \& Topolski, 1995). This pattern was also reported by Paterson et al. (2015) who used a proportional font, but the relationship between number of letters and spatial extent in their investigation for the selected target words was highly linear.

We thus suggest that in situations where the linear relationship between number of letters and spatial extent is intact (monospaced fonts) or highly preserved (the two variables correlate strongly and positively), reporting initial fixation location in terms of letters, only, is appropriate. However, if this linear relationship is decoupled, as in our investigation, the classic initial fixation location findings are only obtained when initial fixation location is reported in spatial, pixel-based measures ${ }^{4}$. This reflects a clear effect of spatial extent, not number of letters, on saccade targeting. Combined with the results of skipping probability and the saccade amplitude measures, we suggest that our findings clearly indicate that saccade targeting measures are influenced by a words' spatial extent.

As for the fixation duration measures (first and single fixation, and gaze durations), our results showing that readers made longer fixations on words that contained more letters, are in line with our hypotheses, and replicate previous findings (in Arabic, Paterson et al., 2015; and other languages, e.g., Hautala et al., 2011; Just \& Carpenter, 1980; McDonald, 2006; Rayner et al., 1996). In addition, the consistent, but non-significant trend in all reported fixation duration measures for slightly increased fixation durations on narrow words compared to wide words probably reflects a modest cost for increased visual crowding in the narrow conditions (see e.g., Bouma, 1970; 1973; Cavanagh, 2001; Drieghe, Brysbaert, \& Desmet, 2005; Loomis, 1978; 
Paterson \& Jordan, 2010; Pelli, Tillman, Freeman, Su, Berger, \& Majaj 2007; and in other writing systems e.g., Chinese: Bai et al., 2008; Zang, Liang, Bai, Yan, \& Liversedge, 2013). As these previous investigations highlighted, the presence of letters in close proximity may reduce the efficiency with which letters are identified, particularly letters that are flanked on both sides with other letters (lateral inhibition, the same applies to strokes in Chinese characters). In our experiment, the modest and non-significant increase in fixation durations appear to correspond to the presence of 5- or 7-letters targets in narrow extent, and do not particularly correspond to the minor differences between the conditions on visual density (or percentage of dark pixels in each word, see above). These very modest increases in fixation durations in narrow extent words, on the other hand, partially correspond with the differences observed between the conditions in terms of the amount of vertical letter overlap reported above (7-Narrow words contained more letter overlap compared to 7-Wide words). Vertical letter overlap may indeed be considered as a more direct source of visual crowding (lateral inhibition). However, perhaps the influence of this visual property of the used font on increasing visual crowding effects was weakened given Arabic readers' over-familiarity with this style of rendering letters (overlapping) in both reading and writing.

Finally, both measures of first pass refixation probability and total fixation count were clearly influenced by a word's spatial extent, not number of letters. Our results thus support the suggestion that due to visual acuity limitations, words that subtend larger spatial extents, regardless of the number of letters, necessitate additional fixations in order to bring the whole word into foveal vision. These results thus clarify previous findings that longer words received more fixations than shorter words in investigations in which the relationship between a word's number of letters and spatial extent was linear (e.g., in Arabic, Paterson et al., 2015; and in other 
European alphabets, Joseph, Liversedge, Blythe, White, \& Rayner, 2009; Just \& Carpenter, 1980; Rayner et al., 1996; and in isolated word reading Vergilino-Perez et al., 2004).

\section{Experiment 2}

The results of Experiment 1 demonstrated that a word's spatial extent, not number of letters influences saccade targeting measures. In our second experiment we aimed to investigate whether initial bigram characteristics also influence saccade targeting. To investigate this, we presented readers with target words that were common Arabic nouns in three conditions: (i) beginning with an initial bigram of extremely high frequency, ال or the; (ii) beginning with a considerably less frequent bigram لل or to/for the, but that is still of relatively high frequency; and (iii) beginning with the initial bigram of the word stem (word stem condition). Average initial bigram frequency in the word stem condition was comparable to that of the bigram to/for the (see below).

To match the target words on the number of letters in the three conditions, targets in the word stem condition were assigned the final bigram ان , which indicates duality (e.g., معلمة, teacher, معلمتان, two teachers). It is important to note that, in terms of word morphology, the initial bigrams ال the and to/for the can be thought of as prefixes, and the final bigram ان لـ for duality can be thought of as a suffix. We had no a priori theoretical reason to expect that this difference in inflectional morphology (the addition of prefixes or suffixes to word stem) would influence saccade targeting in our experiment. The interested reader is referred to evidence from investigations in other Semitic languages such as Hebrew (e.g., Deutsch \& Rayner, 1999). 
Note that both the ال the and لل to/for the initial bigrams are visually similar, and both occupy a narrow extent. This is ideal for comparing these two conditions because they do not differ visually and spatially, but only differ in frequency. Note also that the initial bigrams in the word stem condition, while having an average frequency comparable to لل to/for the condition, features more visually complex letters that occupy wider spatial extent compared to لل to/for the condition. This allowed us to conduct an informative additional contrast between these two conditions to learn whether the differences in their visual/spatial properties had any influence on saccade targeting.

Our first hypothesis was that if saccade targeting was influenced by the frequency of initial bigrams then: (i) readers would make significantly longer saccades and initial fixation location would be considerably closer to word center in the initial bigram condition, compared to the other two conditions; and (ii) means of saccade amplitudes and initial fixation locations should be comparable in the word stem and لل to/for the conditions.

Our second hypothesis was that if any significant differences were obtained between to/for the and the word stem conditions on the measures of saccade targeting, these differences are more likely to result from the visual/spatial differences between these two conditions.

\section{Method}

The participants, apparatus and procedure for this experiment were identical to those in Experiment 1. As explained above, collecting data for both experiments took place in the same session with the stimuli of both experiments acting as filler items for each other. 


\section{Stimuli}

Forty-five sets of target words, 3 words in each set, were created. These target words were embedded in frame sentences that were identical up until the target word. The target word was 6-letters long in 25 sets, and 7-letters in the remaining 20 sets. The spatial extent of 7-letter words was, on average, 9 pixels wider than 6-letter words (6-letter mean spatial extent $=56$ pixels, $\mathrm{SD}=5.9$, range $=41-64 ; 7$-letter mean spatial extent $=65$ pixels, $\mathrm{SD}=6.3$, range $=54-$ 77). This difference in spatial extent was statistically significant $(t(133)=8.6, p<.001)$. Thus, in this experiment, the increase of number of letters was accompanied by an increase of spatial extent of target words $(r=.58)$. Figure 2 illustrates a sample stimulus set. All sentences were written and displayed on a single line and in natural cursive script. The text was rendered in Traditional Arabic font size 18.

$<$ Insert Figure 2 about here $>$

\section{Stimuli matching and norming.}

Initial bigram frequency counts for the three conditions were obtained from the Aralex Corpus (Boudelaa \& Marslen-Wilson, 2010). The frequency of the initial bigram ال the was 68,846.4 per million (PM); لل to/for the was 2,957.7 PM; and average frequency of initial bigrams in the word stem condition was 2,371.1 PM, $\mathrm{SD}=13,86.7$, range $=203.3-5,256.4$. Note that the spatial extent of both ال the and ther the can vary slightly depending on the specific subsequent letter. The spatial extent of the initial bigram, in pixels, for the three 
conditions was as follows: mean ال the $=10.5, \mathrm{SD}=1.3$, range $=8-13$; mean to/for the $=$ 11.2, $\mathrm{SD}=0.7$, range $=10-13 ;$ mean word stem $=17.9, \mathrm{SD}=4$, range $=9-29$. There was a significant difference between the spatial extent of the initial bigrams in the three conditions $(F(2,132)=122.1, p<.001)$. Post hoc analysis using the Tukey HSD test revealed that this difference was due to word stem initial bigrams being significantly wider than in both other conditions $(p \mathrm{~s}<.001)$, whereas there was no difference between the ال the and to/for the initial bigram conditions $(p>0.35)$.

The target words in all three conditions were rated as highly common by 10 subjects who did not take part in the eye tracking procedure (10 ratings per word on a 5-point scale). Average ratings of target word commonness provided by AMTs for each of the conditions were very similar (ال the: mean $=4.03, \mathrm{SD}=0.08$, range $=3.4-4.5$; to/for the: mean $=4.02, \mathrm{SD}=0.08$, range $=3.4-4.4 ;$ word stem $:$ mean $=3.94, \mathrm{SD}=0.10$, range $=3.3-4.5 ; F(2,132)=1.80, p>$ $.15)$.

We made sure that target words in each set had identical spatial extent through extending letter ligatures when necessary. Extending these ligatures would typically increase letters' spatial extent minimally (by a pixel or two) so that all words in a stimulus set would have the spatial extent of the largest word of the set. No ligature extension was performed on the initial bigram of the target words.

Additionally, we obtained 10 cloze predictability ratings for the target word within each sentence. None of the target words in any of the conditions were predictable (produced on zero occasions by the raters). Finally, we obtained 10 ratings as to the naturalness of the sentence structure of all target sentences in all conditions (on a 5-point scale). Sentence structure naturalness ratings for all stimuli were high, with no difference between the conditions (ال the: 
mean $=3.98, \mathrm{SD}=0.10$, range $=3.4-4.6$; to/for the: mean $=3.98, \mathrm{SD}=0.11$, range $=3.5-$ 4.6; word stem $:$ mean $=3.97, \mathrm{SD}=0.10$, range $=3.3-4.6 ; F<1)$.

\section{Design}

The initial bigram of the target words (ال the, to/for the, or word stem) was the withinsubject independent variable. Sentences in these conditions (see sample in Figure 2 Panel A) were counterbalanced using a Latin square, and presented in random order, such that participants saw only one sentence out of each set, and an equal number of target stimuli from all conditions.

\section{Results}

For all reported analyses, we used the same data cleaning criteria described in Experiment 1 . This resulted in removing approximately $1.3 \%$ of all data points. The data cleaning affected all experimental conditions equally (mean number of observations per condition $=533, \mathrm{SD}=2.6$, range $=531-536$ ). Table 5 contains all descriptive statistics for Experiment 2.

$<$ Insert Table 5 about here $>$

Also similar to Experiment 1, we used the lme4 package (same version as above) within the $\mathrm{R}$ environment to run linear mixed models (LMMs). The fixed variables were the conditions of initial bigram of target words. Furthermore, for the sake of completeness, we included the 
number of letters of the target words (6 or 7 letters) as a fixed variable in our models ${ }^{5}$. Subjects and items were treated as the random variables. We always began our analyses with full models (e.g., Barr et al., 2013), and we followed an identical model trimming procedure for the random structure as reported in Experiment 1. For each contrast we report beta values $(b)$, standard error $(S E)$, and $t$ statistics for the saccade targeting measures. Successive differences contrasts were run in which the intercept corresponds to the grand mean and the fixed factor estimates as main effects. Table 6 contains the outputs of the LMMs for these contrasts. We also ran an additional contrast between to/for the and the word stem conditions in order to test the second hypothesis outlined above.

$<$ Insert Table 6 about here $>$

\section{Launch Site}

As Tables 5 and 6 show, there were very small, and non-significant, differences in average launch site between the conditions. Thus, we may assume that any reported effects on initial fixation location are not due to differences in average launch site.

\section{Saccade Amplitude}

As Table 5 shows, differences between saccade amplitudes for the three initial bigram conditions were negligible, with none being statistically significant (see Table 6). The additional contrast revealed that the difference between initial fixation location in the لل to/for the and the 
word stem condition was also not significant $(\mathrm{b}=0.16, \mathrm{SE}=1.16, t=0.14)$. The model output (Table 6) showed that saccade amplitudes differed significantly between 6- and 7-letter words, with saccades being about 7 pixels longer for 7-letter words.

\section{Initial Fixation Location Measured in Letters}

On average, readers landed about 0.2 character further towards the word center in words beginning with ال the compared to لل to/for the condition. This tiny difference was not statistically significant. By contrast, readers landed about 0.7 character further towards word center in words beginning with bigram ال the compared to the word stem initial bigram condition (Table 5) and this difference was significant (see Table 6). Furthermore, the additional contrast revealed that the difference in initial fixation location between لل to/for the and the word stem condition, in which readers landed on average about 0.6 character further into words beginning with لل to/for the bigram was also statistically significant $(\mathrm{b}=0.46, \mathrm{SE}=0.13, t=3.64)$.

We also plotted proportions of fixations landing on each of the letters of the target words (including fixations landing on the space before the first letter). As can be seen in Figure 3, for both 6-and 7-letter words, initial fixation locations on words beginning with the bigrams the and $\mathrm{l}$ to/for the were very similar. However, both these initial bigram conditions differed markedly from the word stem initial bigram condition: In this condition readers targeted a greater proportion of fixations at word beginning, and a smaller proportion near word end, compared to the other two conditions. 
Finally, the results also show that number of letters had a significant effect on initial fixation letter whereby readers landed about 0.5 characters further towards the center in 7 compared to 6-letter words.

\section{Initial Fixation Location as Percentage of Interest Area Extent}

Overall, the differences between the conditions were considerably smaller compared to when initial fixation location was measured in letters. The means obtained (Table 5) suggest that readers were targeting word center in all conditions. This is more in line with the pattern of saccade amplitude reported above. There was no difference between ال the and to/for the conditions. The numerically small difference between words beginning with ال the and 'word stem' bigrams, whereby readers landed about $4 \%$ further towards the center of words starting with ال the approached significance (Table 6). There was no significant effect of number of letters, or interaction with initial bigram conditions. The additional contrast revealed that the difference between initial fixation location in the لل to/for the and the word stem condition was also not significant $(\mathrm{b}=0.18, \mathrm{SE}=1.64, t=0.11)$.

Plotting proportions of fixations landing in $20 \%$ bins of the total pixel extent of the interest area containing the target word revealed a different pattern to that observed for initial fixation locations measured in letters. As can be seen in Figure 4, the trends for increased proportions of fixations on word beginning, and reduced proportions of fixation on word ends in the word stem initial bigram condition is almost entirely absent. Furthermore, the differences between landing distributions for ال the and to/for the initial bigram conditions are minimal. 
The distributions of initial fixation location, measured as a percentage of interest area extent, are thus very similar for all three initial bigram conditions.

$<$ Insert Figure 4 about here $>$

\section{Additional Analyses of Experiment 1 Stimuli}

As our first experiment contained target word sets in which all the words in the set started either with the initial bigram ال the or with the initial bigram of the word stem, we decided to reexamine the data from Experiment 1 to see whether the patterns observed in Experiment 2 would be replicated. We split the stimuli sets of Experiment 1 into two groups, based on the initial bigram of the target word (ال the, or word stem). The full analyses are reported in Appendix 2. The obtained results are very similar to the findings reported in Experiment 2 and can be considered a replication.

\section{Discussion}

In this experiment we aimed to investigate whether saccade targeting could be modulated by the extremely high frequency of the initial bigram ال the in Arabic reading. Essentially, whereas other investigations showed modest influence of very low frequency initial letter strings (see discussion above), we hypothesized that an extreme case such as the initial bigram the may result in a more sizable modulation of saccade targeting. Thus we expected readers to program significantly longer saccades and land significantly further into words beginning with 
the bigram ال the, compared to words beginning with the bigram the, and words beginning with the word stem initial bigram. The contrasts we conducted also allowed us to investigate whether saccade targeting is influenced by the visual/spatial properties of the initial bigrams, given the increased visual complexity and spatial extent of the initial bigrams in the word stem condition.

The results obtained showed that there was no difference between the conditions in terms of launch site. Furthermore, the pattern of saccade amplitudes showed that readers simply targeted word centers, with minimal or no influence of initial bigram characteristics. Rather, saccade amplitudes differed only between the 6- and 7-letter conditions. As mentioned above, previous evidence (Hautala et al., 2011), and the findings from Experiment 1 suggest that words' spatial extent, rather than number of letters, is responsible for the observed modulation of saccade amplitude. Recall that 7-letter words had a wider spatial extent compared to 6-letter words, and we suggest that this is the likely reason for the longer saccades made into 7-letter words. Indeed, in the additional analyses (see Appendix 2) when examining saccade targeting measures on target words from Experiment 1, the results for saccade amplitudes were unequivocal. The only variable that significantly influenced saccade amplitudes was the target words' spatial extent, whereby readers made longer saccades into wider words, with no influence of number of letters, and a minimal influence at most of the initial bigram (see Tables $9 \& 10$ ).

When initial fixation location was measured in letters (i.e., initial fixation letter), there was a minimal difference between ال the the the initial bigram conditions, whereas initial fixation letter in both conditions was significantly closer to word center compared to the word stem condition. This clearly points to a spatial, not linguistic (frequency), influence of initial bigram on initial fixation location given that the initial bigrams were spatially wider in the 
word stem condition compared to the other two conditions. These findings reveal the lack of sensitivity of the saccade targeting system to differences in frequency between relatively high, and extremely high frequency initial bigrams: The presence of extremely high frequency initial bigram did not result in programming significantly longer saccades, nor in significantly shifting initial fixation letter compared to relatively high frequency initial bigrams. These results thus complement existing findings regarding saccade targeting, which showed only modestly influences of initial letter sequences of extremely low frequency (e.g., Hyönä, 1995; White \& Liversedge, 2004; 2006).

As for the reported effects of number of letters on initial fixation location measured in letters (the eyes landed about 0.5 character further into 7 - compared to 6-letter words), we suggest that the most likely cause for this effect is the fact that, as post hoc analyses revealed, 7letter words contained significantly more narrow letters $(\mathrm{l} / \mathrm{a} /, \mathrm{J} / \mathrm{J} /$, and $\mathrm{\rho} / \mathrm{m} /)$, subsequent to the initial bigram, compared to 6-letter words. The average number of narrow letters per word in 6letter words was $1.03, \mathrm{SD}=0.79$; whereas the average for 7 -letter words was $1.5, \mathrm{SD}=0.81$, $t(133)=3.4, p<.001$. This was particularly the case for letter $p / \mathrm{m} /$ (average number of letter ? occurrence per word in 6-letter words $=0.16, \mathrm{SD}=0.37$; average for 7-letter words $=0.43, \mathrm{SD}=$ $0.56, t(133)=3.4, p<.001)$ and particularly as a third letter in the word. This is very likely to have resulted in the initial fixation location corresponding to a letter with a higher ordinal value in 7- compared to 6-letter words.

On the other hand, when initial fixation location was measured as a percentage of the extent of the interest area containing the target word, the difference between the three initial bigram conditions was negligible. This clearly suggests that readers were targeting word centers and the location of the initial fixation was minimally influenced by initial bigram linguistic, or 
spatial, characteristics ${ }^{6}$. We suggest that the discrepancy between the results patterns obtained for the two different measures of initial fixation location can be explained as follows. Firstly, as the results clearly show, readers were targeting word (spatial) centers in all three conditions. Secondly, in the word stem condition, the initial bigram was of a wider extent compared to the other conditions. This meant that the end of letter 2 and beginning of letter 3 were closer to word center in the word stem condition compared to the other two initial bigram conditions (see Figure 2 Panel B). Thus, the pixel at which the initial fixation was made, although close to word center in all three conditions, actually corresponded to a letter with lower ordinal value in the word stem condition compared to the other two initial bigram conditions (ال the and to/for the).

\section{General Discussion}

This study aimed at investigating the influence of a word's number of letters, spatial extent, and initial bigram characteristics on the eye movement control during reading. Arabic was an ideal medium to investigate these effects given its typographical characteristics that allowed us to control for a word's number of letters and spatial extent, using a single font, and without any additional font manipulations. Arabic also features initial bigrams that allowed us to expand on current findings regarding the influence of the frequency of initial letter strings on saccade targeting during reading. Specifically, we anticipated that the extremely high frequency of this initial bigram (ال the) may result in programming longer saccades, and initial fixation locations that land closer to words centers in words beginning with this bigram compared to other words with considerably less frequent initial bigrams. Thus, our findings, in addition to addressing the gaps in our current knowledge about eye guidance in reading of non-European languages, extend what is already known about eye movement control during reading, in general. 
Our results clearly showed that saccade targeting measures (skipping, saccade amplitude, and initial fixation location) are influenced by the upcoming word's spatial extent, and not by the number of letters. This is in line with previous findings (e.g., Hautala et al., 2011). Furthermore, when measured as a percentage of the spatial extent of the interest area containing the target word, the initial fixation location results, along with the saccade amplitude results, fall in line with the classic findings whereby initial fixation location falls closer to word beginning of wide (longer) words, despite longer saccade amplitudes into these words (e.g., McConkie et al. 1988; McConkie et al., 1989; Rayner, 1979; Rayner et al., 1996; Rayner et al., 1998; Vitu et al., 1995). Thus, our findings using a proportional font (also see Hautala et al., 2011) further demonstrate that the classic findings on word length using monospaced fonts reflect the influence of (increasing) spatial extent, and not the number of letters as far as saccade targeting is concerned.

Our findings also revealed that when using proportional fonts that allow letters within a word to subtend unequal amounts of spatial extent, a spatial measure is a more appropriate, and more informative, unit for measuring initial fixation location than a character-based measure. This was apparent from the fact that, in both experiments, changes in initial fixation letters were not accompanied by changes in saccade lengths. In addition, the distributions of initial fixation locations, measured spatially as a percentage of the interest area extent (Figure 4, and Figure 7 in Appendix 2), clearly showed that readers landed slightly before word centers (the preferred viewing location) in all conditions, and in both experiments. Moreover, this appeared to be the case regardless of the degree to which the linear relationship between number of letters and spatial extent is preserved. Recall that in Experiment 1 this relationship was completely decoupled. In Experiment 2 this relationship was somewhat weakened (7-letter words subtended wider extent than 6-letter words, and the correlation between number of letters and spatial extent 
was relatively weaker, $r=.58$, compared to that reported by Paterson et al., 2015, $r=.93$ ). Our findings thus expand upon those of Paterson et al. who documented word length effects in Arabic, but whose stimuli did not allow for a clear distinction to be made between the influences of number of letters and spatial extent on eye movement control during reading.

The results of Experiment 1 show that fixation duration, or the decision of when to move the eyes, is influenced mainly by the number of letters, not spatial extent. Additionally, a consistent pattern of non-significant increases in fixation durations on narrow extent words, relative to wide extent words suggests, at most, a modest cost of visual (Bouma, 1970; 1973; Paterson \& Jordan, 2010) or informational density (e.g., Zang et al., 2013) in the narrow conditions.

With regard to the measures of probability of first pass refixation, and total fixation counts, our results clearly point at the influence of spatial extent, not number of letters. This supports previous suggestions (e.g., Just \& Carpenter, 1980; Rayner et al., 1996; and also in isolated word reading Vergilino-Perez et al., 2004) that visual acuity limitations result in saccade programming that targets refixations at additional locations in wide extent words in order to bring the rest of the letters of a longer word into foveal vision (see also Paterson et al., 2015 for similar findings in Arabic).

As for the influence of initial bigram frequency on saccade targeting, our results were unequivocal. Analyses conducted on Experiment 2 stimuli, which were largely replicated in analyses on Experiment 1 stimuli (Appendix 2), showed clearly that saccade amplitudes were minimally modulated as a function of the frequency of the initial bigrams of the target words. When measured in letters, initial fixation location showed no difference between ال the, the extremely high frequency initial bigram, and to/for the, which is considerably less frequent. 
Indeed, measuring initial fixation location as a percentage of the spatial extent of the interest area clearly showed that readers target word centers, and landed before the word centers in all conditions. Our results thus complement previous findings (e.g., Hyönä, 1995; White \& Liversedge, 2004; 2006) in which saccade targeting was modestly influenced by very low frequency initial letter sequences. In our experiments we clearly show that the saccade targeting system is not overly sensitive to even a large difference in initial bigram frequency of upcoming words, when these initial bigrams are of relatively high, or extremely high, frequency. Coupled with previous findings showing no evidence for modulation of saccade length or initial fixation location because of target words' predictability (e.g., Rayner et al., 2001), or only effects of modest size for initial letter sequence and morphological properties (e.g., Hyönä, 1995; White \& Liversedge, 2004; 2006; Yan et al., 2014), we may conclude that the saccade targeting system is minimally influenced by the linguistic properties of the upcoming words.

To summarize, the findings we reported in this study clearly show that saccade targeting, or the decision of where to move the eyes, is influenced mainly by a word's spatial extent, not number of letters. Additionally, initial bigrams of relatively high, or extremely high frequency have a minimal influence on saccade targeting. Our results also show that the probability of refixation and fixation counts are more influenced by a word's spatial extent, rather than number of letters. Furthermore, fixation duration, or the decision of when to move the eyes, is mainly influenced by the number of letters a word encompasses. These findings thus further illustrate the independence of the when and where decision mechanisms of eye movement control during reading (Rayner \& McConkie, 1976; Rayner \& Pollatsek, 1981). Importantly, our results are obtained using Arabic thus allowing us to expand on the important findings reported by Paterson et al. (2015), particularly with regard to the use of letter-based or spatial measures of initial 
fixation location. Finally, our findings further disentangled the influences of number of letters and spatial extent on eye movement control during reading, in general, making use of the typographical characteristics of Arabic. We furthermore documented, for the first time in reading Arabic, the influence of words' initial bigram characteristics on eye movement control in a way that complemented existing results from other alphabetic languages. 


\section{References}

Abu-Rabia, S. (2007). The role of morphology and short vowelization in reading Arabic among normal and dyslexic readers in grades 3, 6, 9, and 12. Journal of Psycholinguistic Research, 36, 89-106. doi:10.1007/s10936-006-9035-6

Baayen, R. H., Davidson, D. J., \& Bates, D. M. (2008). Mixed-effects modelling with crossed random effects for subjects and items. Journal of Memory and Language, 59, 390-412. doi:10.1016/j.jml .2007.12.005

Barr, D. J., Levy, R., Scheepers, C., \& Tily, H. J. (2013). Random effects structure for confirmatory hypothesis testing: Keep it maximal. Journal of Memory and Language, 68, 255-278. doi:10.1016/j.jml .2012.11.001

Bates, D., Maechler, M., \& Bolker, B. (2011). Lme4: Linear mixed-effects models using S4 classes. R package version 0.999375-42 [Computer software]. Avail- able from $\underline{\text { http://CRAN.R-project.org/package=lme4 }}$

Bai, X., Yan, G., Liversedge, S. P., Zang, C., \& Rayner, K. (2008). Reading spaced and unspaced Chinese text: Evidence from eye movements. Journal of Experimental Psychology: Human Perception and Performance, 34, 1277-1287. doi:10.1037/00961523.34.5.1277

Beauvillain, C., \& Doré, K. (1998). Orthographic codes are used in integrating information from the parafovea by the saccadic computation system. Vision Research, 38, 115-123. doi:10.1016/S0042-6989(97)00127-2

Beanvillain, C., Doré, K., \& Baudouin, V. (1996). The "center of gravity" of words: Evidence for an effect of the word-initial letters. Vision Research, 36, 589-603. doi:10.1016/00426989(95)00141-7 
Bertram, R., \& Hyönä, J. (2003). The length of a complex word modifies the role of morphological structure: Evidence from eye movements when reading short and long Finnish compounds. Journal of Memory and Language, 48, 615-634. doi:10.1016/S0749596X(02)00539-9

Boudelaa, S., \& Marslen-Wilson, W. D. (2010). Aralex: A lexical database for Modern Standard Arabic. Behavior Research Methods, 42, 481-487. doi:10.3758/BRM.42.2.481

Bouma, H. (1970). Interaction effects in parafoveal letter recognition. Nature, 226, 177-178. doi:10.1038/226177a0

Bouma, H. (1973). Visual interference in the parafoveal recognition of initial and final letters of words. Vision Research, 13, 767-782. doi:10.1016/0042-6989(73)90041-2

Brysbaert, M., Drieghe, D., \& Vitu, F. (2005). Word skipping: Implications for theories of eye movement control in reading. In G. Underwood (Ed.), Cognitive processes in eye guidance. Oxford, UK: Oxford University Press.

Calvo, M. G., \& Meseguer, E. (2002). Eye movements and processing stages in reading: Relative contributions of visual, lexical, and contextual factors. Spanish Journal of Psychology, 5, 66-77. doi:10.1017/S1138741600005849

Cavanagh, $P$. (2001). Seeing the forest but not the trees. Nature Neuroscience, 4, 673-674. doi: $10.1038 / 89436$

Deutsch, A., \& Rayner, K. (1999). Initial fixation location effects in reading Hebrew words. Language and Cognitive Processes, 14, 393-421. doi:10.1080/016909699386284

Doré, K., \& Beauvillain, C. (1997). Latency dependence of word-initial letter integration by the saccadic system. Perception \& Psychophysics, 59, 523-533. doi:10.3758/BF03211861 
Drieghe, D., Brysbaert, M., \& Desmet, T. (2005). Parafoveal-on-foveal effects on eye movements in text reading: Does an extra space make a difference? Vision Research, 45, 1693-1706. doi:10.1016/j.visres.2005.01.010

Drieghe, D., Brysbaert, M., Desmet, T., \& De Baecke, G. (2004). Word skipping in reading: On the interplay of linguistic and visual factors. European Journal of Cognitive Psychology, 16, 79-103. doi:10.1080/09541440340000141

Drieghe, D. (2008). Foveal processing and word skipping during reading. Psychonomic Bulletin \& Review, 15, 856-860. doi:10.3758/PBR.15.4.856

Ehrlich, S. F., \& Rayner, K. (1981). Contextual effects on word perception and eye movements during reading. Journal of Verbal Learning and Verbal Behavior, 20, 641-655. doi:10.1016/S0022-5371(81)90220-6

Frost, R., Kugler, T., Deutsch, A., \& Forster, K. (2005). Orthographic structure versus morphological structure: Principles of lexical organization in a given language. Journal of Experimental Psychology: Learning, Memory, and Cognition, 31, 1293-1326. doi:10.1037/0278-7393.31.6.1293

Hautala, J., Hyönä, J., \& Aro, M. (2011). Dissociating spatial and letter-based word length effects observed in readers' eye movement patterns. Vision Research, 51, 1719-17-27. doi:10.1016/j.visres.2011.05.015

Hyönä, J. 1993). Eye movements during reading and discourse processing [Psychological Research Rep. No. 65]. Turku, Finland: University of Turku.

Hyönä, J. (1995). Do irregular letter combinations attract readers' attention? Evidence from fixation locations in words. Journal of Experimental Psychology: Human Perception and Performance, 2, 68-81. doi:10.1037/0096-1523.21.1.68 
Hyönä, J., \& Olson, R. K. (1995). Eye fixation patterns among dyslexic and normal readers: Effects of word length and word frequency. Journal of Experimental Psychology: Learning, Memory, and Cognition, 21, 1430-1440. doi:10.1037/0278-7393.21.6.1430

Inhoff, A. W., Radach, R., Eiter, B., \& Juhasz, B. (2003). Distinct subsystems for the parafoveal processing of spatial and linguistic information during eye fixations in reading. Quarterly Journal of Experimental Psychology: Human Experimental Psychology, 56A, 803-828. doi:10.1080/02724980244000639

Joseph, H. S. S. L., Liversedge, S. P., Blythe, H. I., White, S. J., \& Rayner, K. (2009). Word length and landing position effects during reading in children and adults. Vision Research, 49, 2078-2086. doi:10.1016/j.visres.2009.05.015

Juhasz, B. J., White, S. J., Liversedge, S. P., \& Rayner, K. (2008). Eye movements and the use of parafoveal word length information in reading. Journal of Experimental Psychology: Human Perception and Performance, 34, 1560-1579. doi:10.1037/a0012319)

Just, M. A., \& Carpenter, P. A. (1980). A theory of reading: From eye fixations to comprehension. Psychological Review, 87, 329-354. doi:10.1037/0033-295X.87.4.329

Li, X., \& Shen, W. (2013). Joint effect of insertion of spaces and word length in saccade target selection in Chinese reading. Journal of Research in Reading, 36, s64-s77. doi:10.1111/j.1467-9817.2012.01552.x

Liu, P., Li, W., Han, B., \& Li, X. (2014). Effects of anomalous and small stroke omissions on eye movements during the reading of Chinese sentences. Ergonomics. doi:10.1080/00140139.2014.945492

Loomis, J. M. (1978). Lateral masking in foveal and eccentric vision. Vision Research, 18, 335338. doi:10.1016/0042-6989(78)90168-2 
Matin, E. (1974). Saccadic suppression: A review and analysis. Psychological Bulletin, 81, 899-917. doi:10.1037/h0037368

McConkie, G. W., Kerr, E. W., \& Dyre, B. E. (1994). What are "normal" eye movements during reading: Toward a mathematical description. In J. Ygge \& G. Lennerstrand (Eds.), Eye movements in reading (pp. 315-328). Oxford, England: Pergamon Press.

McConkie, G. W., Kerr, E W., Reddix, M. D., \& Zola, D. (1988). Eye movement control during reading: I. The location of initial eye fixations in words. Vision Research, 28, 1107-1118. doi:10.1016/0042-6989(88)90137-X

McConkie, G. W., Kerr, P. W., Reddix, M. D., Zola, D., \& Jacobs, A. M. (1989). Eye movement control during reading: II. Frequency of refixating a word. Perception \& Psychophysics, 46, 245-253. doi:10.3758/BF03208086

McDonald, S. A. (2006). Effects of number-of-letters on eye movements during reading are independent from spatial word length. Visual Cognition, 13, 89-98. doi:10 1080113506280500143367

Morris, R. K., Rayner, K., \& Pollatsek, A. (1990). Eye movement guidance in reading: The role of parafoveal letter and space information. Journal of Experimental Psychology: Human Perception and Performance, 16, 268-281. doi:10.1037/0096-1523.16.2.268

Nuthmann, A., Engbert, R., \& Kliegl, R. (2005). Mislocated fixations during reading and the inverted optimal viewing position effect. Vision Research, 45, 2201-2217. doi:10.1016/j.visres.2005.02.014

O’Regan, J. K., \& Lévy-Schoen, A. (1983). Integrating visual information from successive fixations: Does trans-saccadic fusion exist? Vision Research, 23, 765-768. doi:10.1016/0042-6989(83)90198-0 
Paterson, K. B., Almabruk, A. A. A., McGowan, V. A., White, S. J., \& Jordan, T. J. (2015). Effects of word length on eye movements control: The evidence from Arabic. Psychonomic Bulletin \& Review. doi: 10.3758/s13423-015-0809-4

Paterson, K. B., \& Jordan, T. R. (2010). Effects of increased letter spacing on word identification and eye guidance during reading. Memory \& Cognition, 38, 502-5012. doi:10.3758/MC.38.4.502

Pelli, D. G., Tillman, K. A., Freeman, J., Su, M., Berger, T. D., and Majaj, N. J. (2007). Crowding and eccentricity determine reading rate. Journal of Vision, 7, 20-36. doi: $10.1167 / 7.2 .20$

Plummer, P., 7 Rayner, K. (2012). Effects of parafoveal word length and orthographic features on initial fixation landing positions in reading. Attention, Perception, and Psychophysics, 74, 950-963. doi:10.3758/s13414-012-0286-Z

Pollatsek, A., \& Rayner, K. (1982). Eye movement control in reading: The role of word boundaries. Journal of Experimental Psychology: Human Perception and Performance, 8, 817-833. doi:10.1037/0096-1523.8.6.817

R Core Team. (2013). A language and environment for statistical computing. Vienna, Austria: R Foundation for Statistical Computing. http:// www.R-project.org/

Radach, R., Inhoff, A., \& Heller, D. 2004). Orthographic regularity gradually modulates saccade amplitudes in reading. European Journal of Cognitive Psychology, 16, 27-51. doi:10.1080/09541440340000222

Radach, R., \& McConkie, G. W. (1998). Determinants of fixation positions in words during reading. In G. Underwood (Ed.), Eye guidance in reading and scene perception (pp. 77101). Oxford, England: Elsevier. 
Rayner, K. (1975). The perceptual span and peripheral cues in reading. Cognitive Psychology, 7, 65-81. doi:10.1016/0010-0285(75)90005-5

Rayner, K. (1979). Eye guidance in reading: Fixation location with words. Perception, 8, 21 30. doi: $10.1068 / \mathrm{p} 080021$

Rayner, K., Binder, K. S., Ashby, J., \& Pollatsek, A. (2001). Eye movement control in reading: Word predictability has little influence on initial landing positions in words. Vision Research, 41, 943-954. doi:10.1016/S0042-6989(00)00310-2

Rayner, K. (2009). Eye movements and attention in reading, scene perception, and visual search. The Quarterly Journal of Experimental Psychology, 62, 1457-1506. doi:10.1080/17470210902816461

Rayner, K., Fischer, M. H., \& Pollatsek, A. (1998). Unspaced text interferes with both word identification and eye movement control. Vision Research, 38, 1129-1144. doi:10.1016/S0042-6989(97)00274-5

Rayner, K., \& McConkie, G. W. (1976). What guides a reader's eye movements? Vision Research, 16, 829-837. doi:10.1016/0042-6989(76)90143-7

Rayner, K., \& Pollatsek, A. (1981). Eye movement control during reading: Evidence for direct control. Quarterly Journal of Experimental Psychology, 33A, 351-373. doi:10.1080/14640748108400798

Rayner, K., \& Pollatsek, A. (1996). Reading unspaced text is not easy: Comments on the implications of Epelboim et al.'s study for models of eye movement control in reading. Vision Research, 36, 461-470. doi:10.1016/0042-6989(95)00132-8 
Rayner, K., Sereno, S. C., \& Raney, G. E. (1996). Eye movement control in reading: A comparison of two types of models. Journal of Experimental Psychology: Human Perception and Performance, 22, 1188-1200. doi:10.1037/0096-1523.22.5.1188

Rayner, K., Slattery, T. J., \& Bélanger, N. N. (2010). Eye movements, the perceptual span, and reading speed. Psychonomic Bulletin \& Review, 17, 834-839. doi:10.3758/PBR.17.6.834

Rayner, K., \& Well, A. D. (1996). Effects of contextual constraint on eye movements in reading: a further examination. Psychonomic Bulletin \& Review, 3, 504-509. doi:10.3758/BF03214555

Vainio, S., Hyönä, J., \& Pajunen, A. (2009). Lexical predictability exerts robust effects on fixation duration, but not on initial landing position during reading. Experimental Psychology, 56, 66-74. doi:10.1027/1618-3169.56.1.66

Vergilino-Perez, D., Collins, T., \& Doré-Mazars, K. (2004). Decision and metrics of refixations in reading isolated words. Vision Research, 44, 2009-2017.

doi:10.1016/j.visres.2004.03.012

Vitu, E, O'Regan, J. K., Inhoff, A. W., \& Topolski, R. (1995). Mindless reading: Eye movement characteristics are similar in scanning letter strings and reading text. Perception \& Psychophysics, 57, 352-364. doi:10.3758/BF03213060

Vitu, F., O’Regan, J. K., \& Mittau, M. (1990). Optimal landing position in reading isolated words and continuous text. Perception \& Psychophysics, 47, 583-600. doi:10.3758/BF03203111

Vonk, W., Radach, R., \& van Rijn, H. 2000). Eye guidance and the saliency of word beginnings in reading text. In A. Kennedy, R. Radach, D. Heller, \& J. Pynte (Eds.), Reading as a perceptual process (pp. 269-299. Oxford, UK: Elsevier. 
White, S. J., \& Liversedge, S. P. (2004). Orthographic familiarity influences initial eye positions in reading. European Journal of Cognitive Psychology, 16, 52-78. doi:10.1080/09541440340000204

White, S. J., \& Liversedge, S. P. (2006). Linguistic and nonlinguistic influences on the eyes' landing positions during reading. Quarterly Journal of Experimental Psychology, 59, 760782. doi:10.1080/02724980543000024

White, S. J., Rayner, K., \& Liversedge, S. P. (2005). The influence of parafoveal word length and contextual constraint on fixation durations and word skipping in reading. Psychonomic Bulletin \& Review, 12, 466-471. doi:10.3758/BF03193789

Wightwick, J., \& Gaafar, M. (2005). Mastering Arabic script. London: g-and-w Publishing. Zang, C., Liang, F., Bai, X, \& Liversedge, S. P. (2013). Interword spacing and landing position effects during Chinese reading in children and adults. Journal of Experimental Psychology: Human Perception and Performance, 39, 720-734. doi:10.1037/a0030097 


\section{Footnotes}

${ }^{1}$ We actually created 40 sets of stimuli, and the participants saw stimuli from all 40 sets. However due to an error in matching the words in 3 sets on the presence or absence of the initial bigram ال or the, we excluded these three sets from all analyses reported.

${ }^{2}$ We performed additional analyses in which the amount of vertical letter overlaps was included in linear mixed models as another fixed variable. The results indicated that the amount of vertical letter overlaps did not influence any of the saccade targeting measures.

${ }^{3}$ In Figure 1 Panel C, only narrow extent 5- and 7-letter examples are illustrated, however the same observation applies equally to wide extent words: Fixating the same location, around the center of the target word's spatial extent, results in fixating the second letter in 5Wide words, and the third letter in 7-Wide words.

${ }^{4}$ Distributions of initial fixation locations on letters, and on bins of $20 \%$ increments of the spatial extent of the interest area, clearly support these suggestions. Illustrations and discussion of these distributions are available in Appendix 2, where we re-examine saccade targeting measures as part of the investigation reported in Experiment 2.

${ }^{5}$ For all the saccade targeting measures we report, we obtained almost identical effects to those reported here when we collapsed across number of letters.

${ }^{6}$ The results for both the saccade amplitude and initial fixation location measures can also be considered as a demonstration that inflectional morphology (the presence of prefixes vs. suffixes) does not influence saccade targeting in Semitic languages (Arabic). See Deutsch and Rayner (1999) for further discussion. 


\section{Appendix 1}

\section{Font Selection}

We presented 15 native Arabic readers (Amazon Mechanical Turkers) with short passages of text (about 45 words long), rendered in proportional fonts (Arial, Times New Roman, Traditional Arabic, Lateef, and Scheherazade), and monospaced fonts (Courier New, Simplified Arabic Fixed, and Thabit, see Figure 5). We asked the participants to rate the naturalness of the look and clarity of text rendered in each font. Specifically, naturalness of look was used as a subjective measure of how the passage of text appears to the reader, whereas clarity was a subjective measure of how easily identifiable are letters' and words' features in each of the fonts. The participants were also asked to report an estimate of the regularity with which they encounter these fonts. To be explicit, in asking participants to report such an estimate, we did not make the assumption that they knew the name of the font they were looking at. Rather, we asked readers to simply report how often the texts they regularly read appeared visually similar to the sample texts of each of the fonts they were comparing.

$<$ Insert Figure 5 about here $>$

The readers used a 7-point scale to provide their ratings: 1 = text looking very unnatural / very unclear / rarely encountered in reading; and $7=$ text looking perfectly natural / perfectly clear / very often encountered in reading. In addition, the participants were also asked to briefly comment on what makes the text look natural / clear or less so. 
As Table 7 indicates, participants rated both the Traditional Arabic and Scheherazade fonts as more natural looking, and clearer than the other proportional fonts (all $p \mathrm{~s}<.01$ ). Traditional Arabic and Scheherazade however did not differ significantly on these two measures $(p s>9)$. Also, the five proportional fonts that were compared did not differ significantly from each other on the measure of how frequently they are encountered by readers $(F(4,70)=1.3, p<$ .3). Given that the Traditional Arabic font had the highest means in all measures, we decided to use traditional Arabic in the current investigation.

$<$ Insert Table 7 about here $>$

Although we do not use monospaced fonts in the current investigation, we sought to obtain some norming data concerning readers' perception of the visual properties of Arabic text rendered in monospaced fonts, compared to the ubiquitous proportional fonts. Thus, comparing proportional and monospaced fonts, as Table 1 shows, the participants indicated unequivocally that the text rendered in monospaced fonts (Courier New, Simplified Arabic Fixed, and Thabit) looked considerably less natural $(t(118)=15.2, p<.001)$, and less clear $(t(118)=17, p<.001)$ compared to proportional fonts. The participants also indicated that monospaced fonts are considerably encountered less frequently during reading compared to proportional fonts $(t(118)=$ $28.9, p<.001)$. Furthermore, participants commented that in monospaced fonts words appear unnecessarily large. Specifically, given that in monospaced fonts the size of the horizontal ligature (lines) is increased in many characters to render all characters of equal spatial extent, words appear larger in monospaced fonts (e.g., compare the size of the horizontal ligatures, in الـــ اللـون or color in Times New Roman or Arial fonts, and in Courier New font 
in Figure 5). In addition to changing the size of the ligatures, the identifying characteristics in the letters are rendered much reduced in monospaced fonts, particularly in the Thabit and Simplified Arabic Fixed fonts (e.g., the top portions of letters such as $9 /$ w/, making it look more

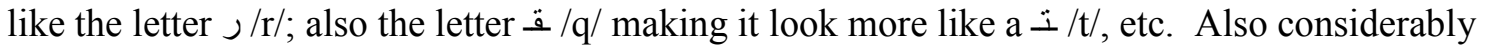
narrowing letters with upward-curving descenders at the end of words such as ض ص شس). Thus, monospaced fonts were rated considerably less clear and natural. 


\section{Appendix 2}

\section{Additional Analyses for Experiment 2: Saccade Targeting Measures in Experiment 1 Stimuli as Function of Initial Bigram}

In Experiment 1 we selected either 5- or 7-letter target words that subtended identical spatial extents that were either narrow or wide. Additionally, the target words used in this experiment, in 15 of the 37 target sets, started with the initial bigram of the word stem, and in the remaining 22 sets the target words began with the bigram ال the. Thus, we used these stimuli to further examine the effect of the initial bigram frequency on saccade targeting. As mentioned above, according to the Aralex corpus (Boudelaa \& Marslen-Wilson, 2010), the frequency of the initial bigram ال the is 68,846.4 per million. Collapsed across number of letter and spatial extent conditions, average frequency for initial bigrams in the word stem condition was 2585.1 PM, SD $=3740.8$, range $=2.61-14,216.6$ (see Table 8 for word stem initial bigram frequencies for each of the number of letters and spatial extent conditions). Collapsed across number of letter and spatial extent conditions, the average spatial extent of the initial bigram, in pixel, for lise as the initial bigram was $9.5(\mathrm{SD}=1.9$, range $=7-13)$ and for the word stem initial bigram was 15.9 $(\mathrm{SD}=5.9$, range $=9-22$, see Table 8 for the and word stem initial bigram spatial extents for each of the number of letters and spatial extent conditions). Thus, similar to Experiment 2 ال stimuli, initial bigrams had a significantly wider extent in the word stem condition compared to the condition $(t(146)=8.9, p<.001)$.

$<$ Insert Table 8 about here $>$ 
For the saccade targeting measures reported below, we added initial bigram as a fixed variable (with two levels: ال the vs. 'word stem') to the LMMs, in addition to number of letters (5 vs. 7), and spatial extent (narrow vs. wide). Furthermore, model trimming followed the same procedure described in Experiment 1. For all reported analyses, Table 9 contains the descriptive statistics, Table 10 contains the outputs of the LMMs for the saccade targeting measures.

\author{
$<$ Insert Table 9 about here $>$ \\ $<$ Insert Table 10 about here $>$
}

\title{
Launch Site
}

As Tables 9 and 10 show, there were very small, and non-significant, differences in average launch site for all conditions.

\section{Saccade Amplitude}

Readers made saccades that were numerically longer ( 4.14 pixels, about $0.17^{\circ}$ of visual angle) into words beginning with ال the relative to words beginning with the word stem initial bigram. This difference approached significance (see Table 10).

There were no effects for number of letters on saccade amplitude. There was however a significant effect of words' spatial extent such that readers made saccades that were about 3 pixels (about $0.12^{\circ}$ of visual angle) longer into wide words relative to narrow words. There were no significant interactions. 


\section{Initial Fixation Location Measured in Letters}

As can be seen in Table 9, readers landed about 0.5 character further into target words beginning with bigram ال the compared to target words beginning with the initial bigram of the word stem. This difference was statistically significant (Table 10) and in line with the results from Experiment 2.

We plotted proportions of fixations landing on each of the letters of the target words (including fixations landing on the space before the first letter). As can be seen in Figure 6, the peak of landing distributions shifted further towards word center for words beginning with the bigram, compared to the word stem initial bigram. Furthermore, the landing distributions show that readers targeted a relatively greater proportion of fixations at the word beginning, and a smaller proportion near word end, in the word stem initial bigram condition compared to the.

$<$ Insert Figure 6 about here $>$

Similar to what we reported in Experiment 1, there was a significant effect of number of letters on initial fixation letter, in the same direction and of similar magnitude to the analyses reported above. Furthermore, there was no significant interaction between number of letters and initial bigram characteristics of the target.

Initial Fixation Location as Percentage of Interest Area Extent 
There was no effect of initial bigram on initial fixation location measured as a percentage of the interest area extent. The numerical differences between the two initial bigram conditions suggested that readers landed further into words beginning with the bigram ال the but these differences were small and non-significant.

The only significant effect on this spatial measure of initial fixation location was that of a target word's spatial extent whereby, similar to what was reported in Experiment 1, readers landed further into narrower words, relative to wider words. There was no effect of number of letters, or any significant interactions (see Tables $9 \& 10$ ).

Plotting proportions of fixations landing in $20 \%$ bins of the total pixel extent of the interest area containing the target word revealed a different pattern to that observed when initial fixation location was measured in letters. As can be seen in Figure 7, the trends observed in initial landing letter, namely, shifted distribution peak towards word beginning, increased proportions of fixations on word beginning, and reduced proportions of fixation on word ends in the word stem initial bigram condition, are largely absent. Rather, very similar landing distribution patterns are observed between the conditions based on their spatial extent, with narrow conditions patterning similarly, while differing from wide conditions. 


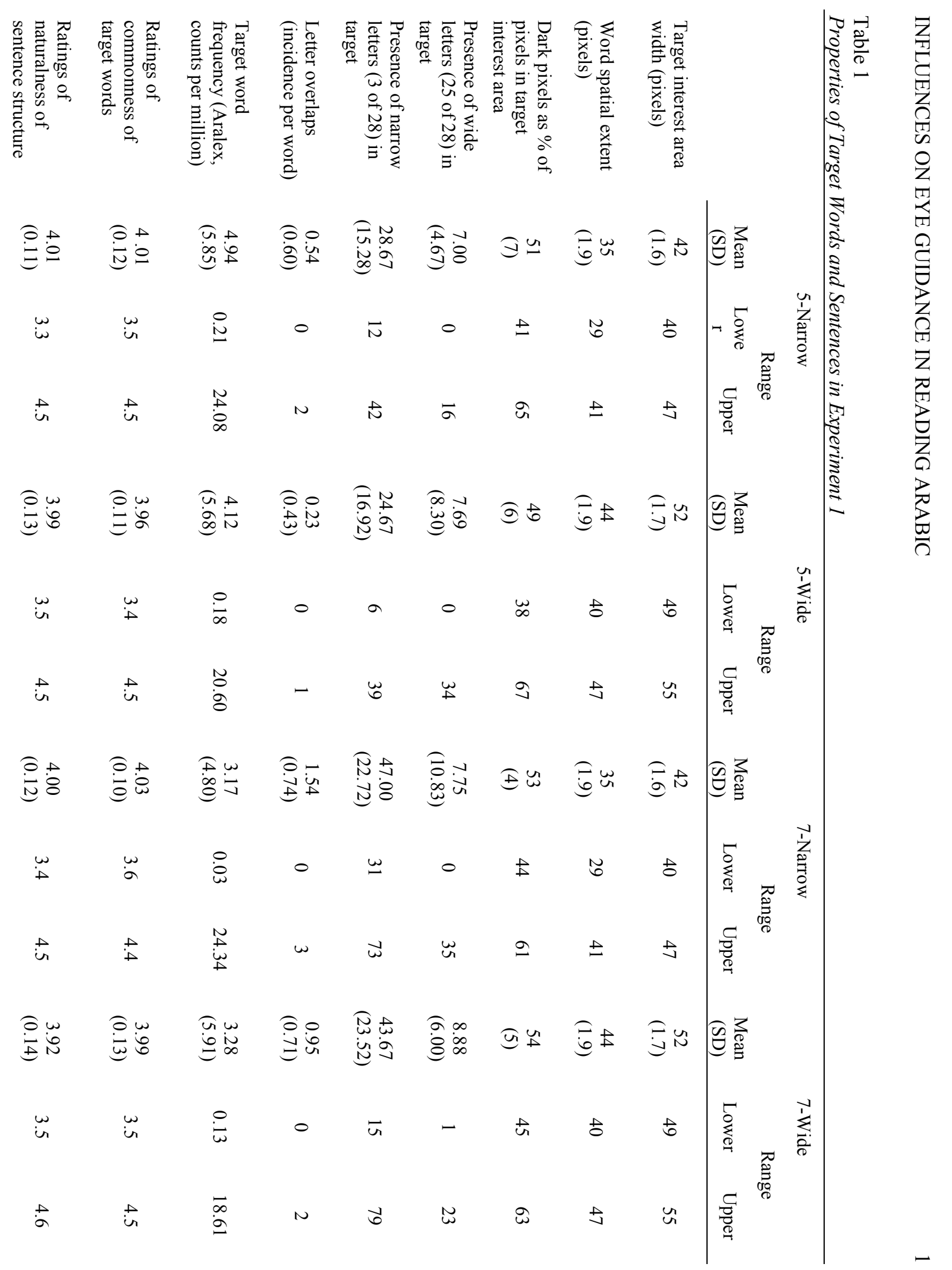


Table 2

Eye Movement Measures Reported for Experiment 1

\begin{tabular}{lcccc}
\hline & 5-Narrow & 5-Wide & 7-Narrow & 7-Wide \\
& Mean (SD) & Mean (SD) & Mean (SD) & Mean (SD) \\
\cline { 2 - 5 } Launch site (pixel*) & $27.65(22.29)$ & $26.42(18.26)$ & $26.43(21.57)$ & $26.55(18.67)$ \\
Skipping probability & $0.08(0.27)$ & $0.03(0.18)$ & $0.08(0.28)$ & $0.02(0.14)$ \\
Saccade amplitude (pixel*) & $48.58(20.34)$ & $50.89(16.02)$ & $47.34(20.43)$ & $50.78(16.12)$ \\
First fixation letter & $2.57(1.57)$ & $2.49(1.50)$ & $3.07(2.12)$ & $3.07(2.00)$ \\
First fixation location as \% of & $49.49(26.47)$ & $47.09(24.21)$ & $49.83(26.42)$ & $46.74(24.92)$ \\
IA extent & $319(160.21)$ & $293(121.03)$ & $321(143.27)$ & $316(154.59)$ \\
First fixation duration (ms) & $326(158.82)$ & $306(107.78)$ & $344(149.63)$ & $332(164.00)$ \\
Single fixation duration (ms) & $413(241.34)$ & $403(204.59)$ & $443(295.52)$ & $427(250.54)$ \\
Gaze duration (ms) & $0.31(0.46)$ & $0.40(0.49)$ & $0.29(0.45)$ & $0.34(0.48)$ \\
First pass refixation probability & $1.85(1.19)$ & $2.16(1.42)$ & $1.97(1.33)$ & $2.21(1.40)$ \\
\hline Total fixation count & & &
\end{tabular}

Note. $*$ Average letter size $=7.7$ pixels 


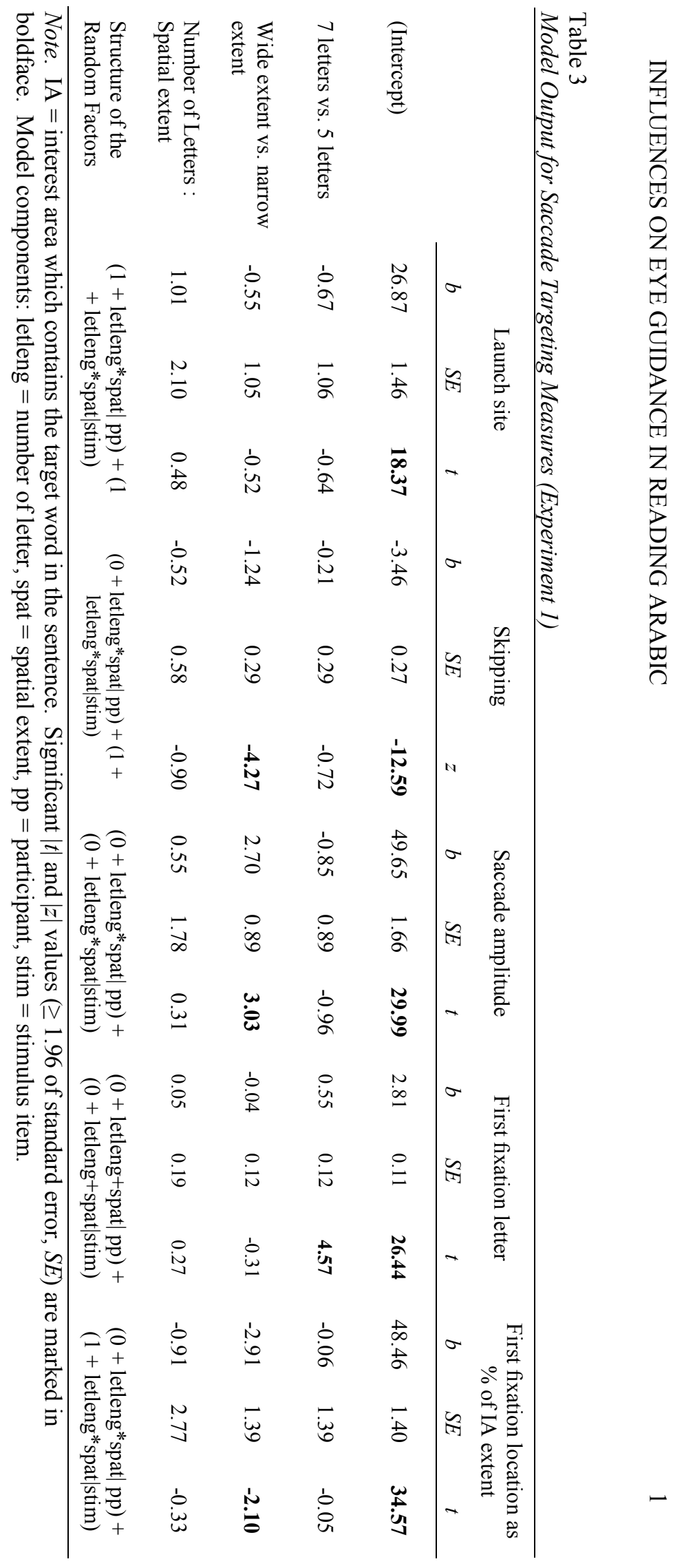




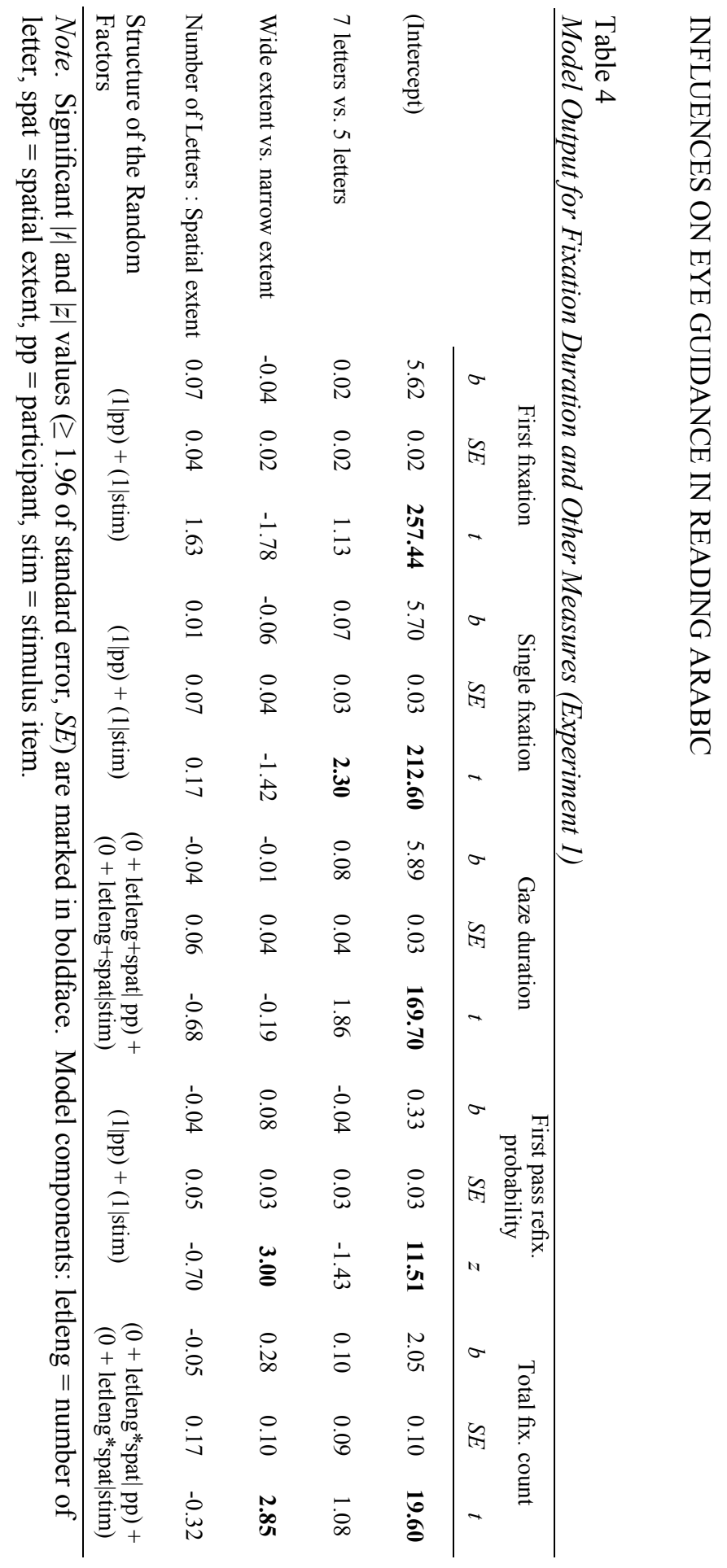


Table 5

Saccade Targeting Measures Reported in Experiment 2

\begin{tabular}{lcccc}
\hline & $\begin{array}{c}\text { Number } \\
\text { of } \\
\text { Letters }\end{array}$ & Mean (SD) & WFor/To The & Word Stem \\
\cline { 2 - 4 } & 6 & $26.31(18.60)$ & $27.66(21.00)$ & Mean (SD) \\
Launch site (pixel) & 7 & $24.38(18.28)$ & $25.50(20.12)$ & $27.07(27.10)$ \\
& 6 & $46.84(11.91)$ & $45.99(11.24)$ & $44.98(12.61)$ \\
Saccade amplitude (pixel) & 7 & $52.63(13.17)$ & $52.13(13.10)$ & $51.10(13.13)$ \\
Initial fixation letter & 6 & $3.35(1.72)$ & $3.13(1.78)$ & $2.64(1.67)$ \\
Initial fixation location as & 6 & $3.76(1.90)$ & $3.69(2.00)$ & $3.08(1.73)$ \\
\% of IA extent & 7 & $51.31(23.43)$ & $50.42(21.80)$ & $48.55(19.92)$ \\
\hline
\end{tabular}

Note. Average character size $=7.7$ pixels. 


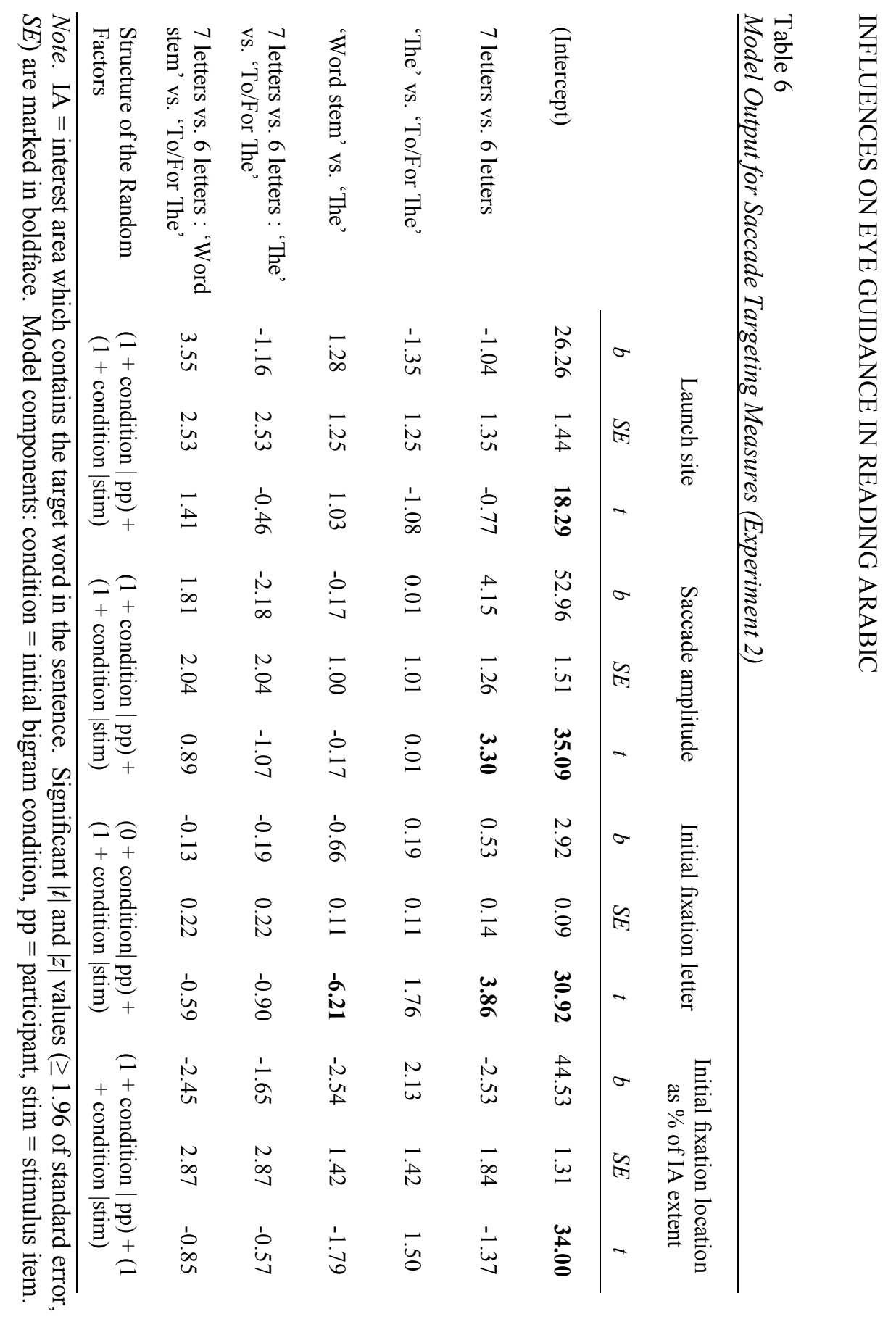




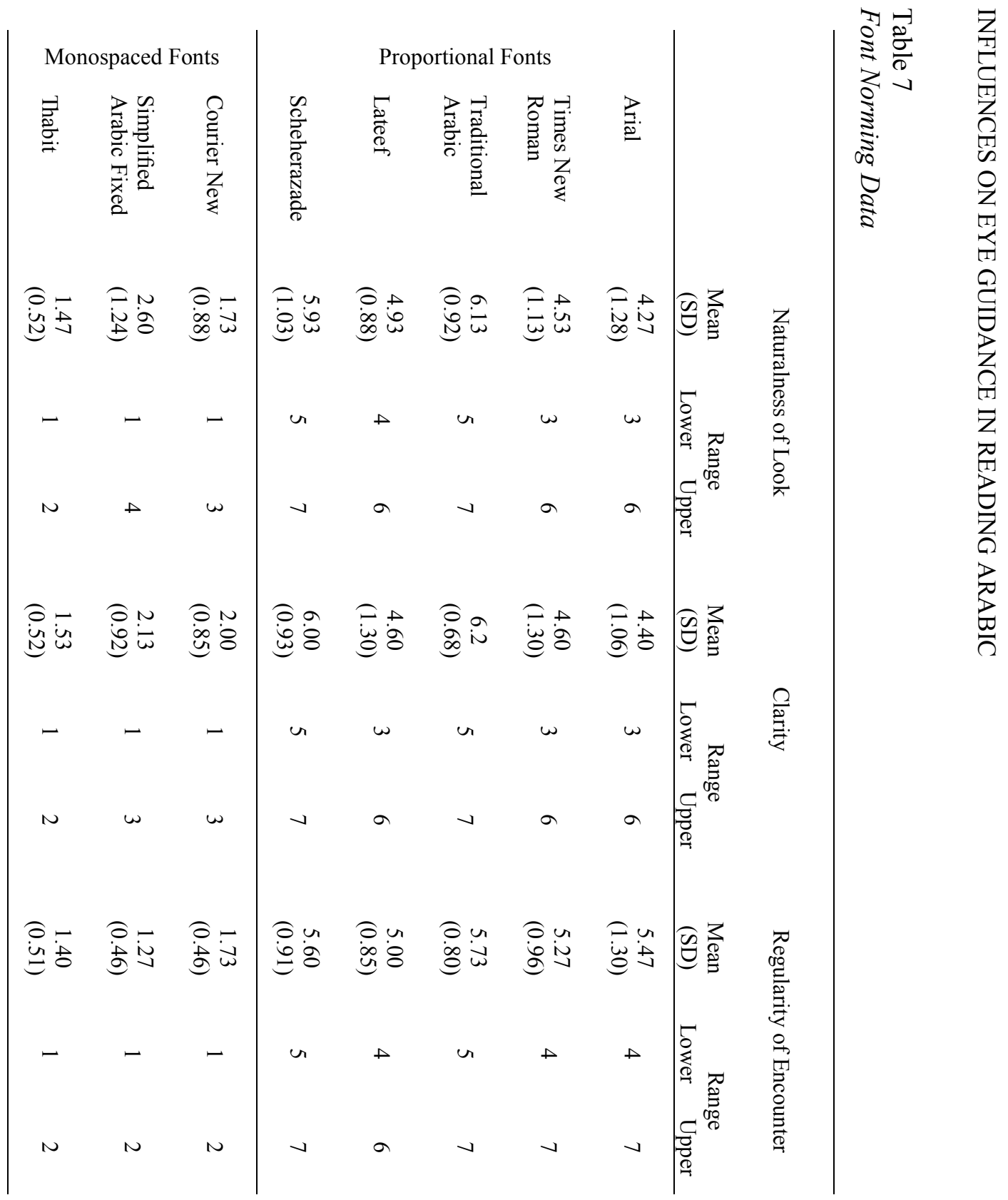




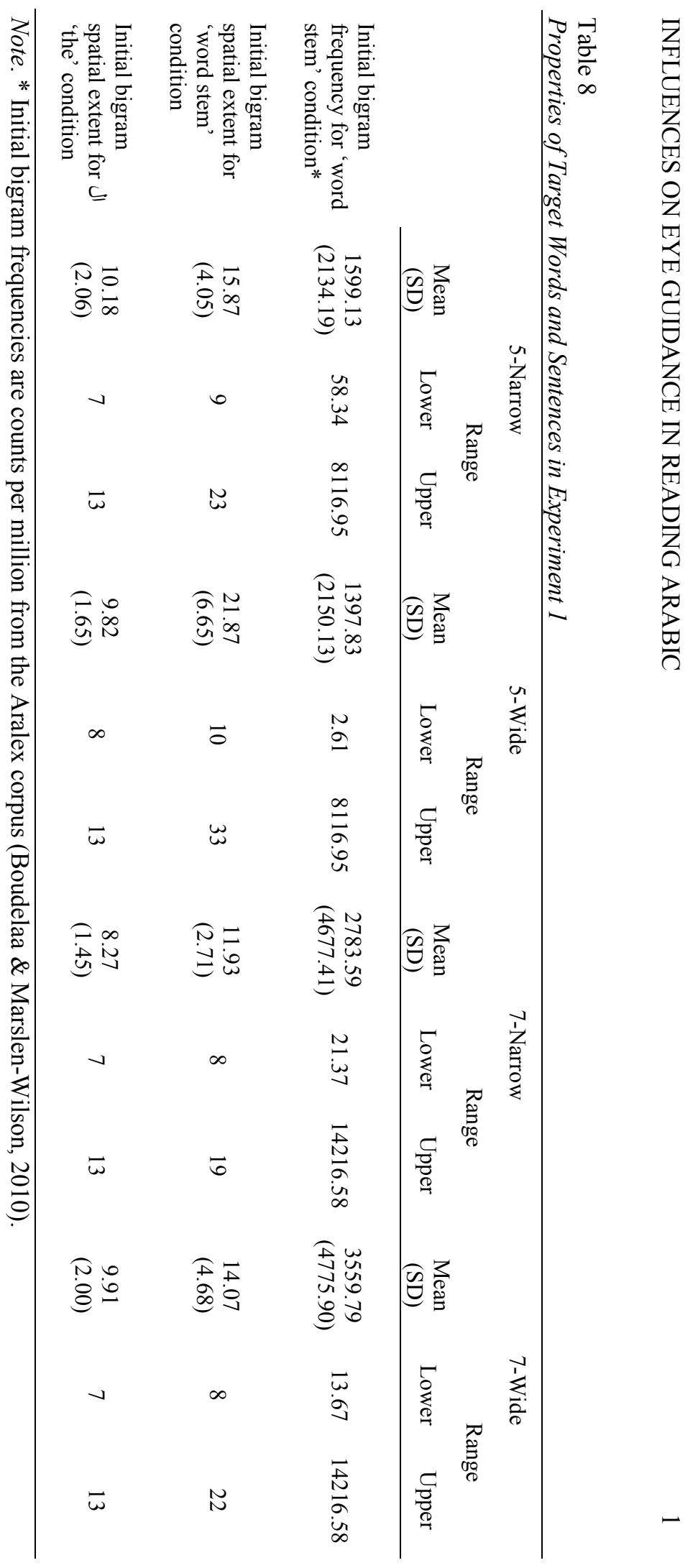


Table 9

Additional Saccade Targeting Measures for Experiment 1

5-Narrow 7-Wide 7-Narrow

\begin{tabular}{llllll} 
& \multicolumn{1}{c}{$\begin{array}{c}\text { Initial } \\
\text { Bigram } \\
\text { Condition }\end{array}$} & Mean (SD) & Mean (SD) & Mean (SD) & Mean (SD) \\
\cline { 2 - 6 } & The & $27.30(22.38)$ & $26.46(17.93)$ & $28.96(23.61)$ & $28.38(19.36)$ \\
Launch site (pixel) & Word stem & $25.25(22.03)$ & $26.37(18.8)$ & $26.66(17.55)$ & $26.90(24.35)$ \\
& The & $50.37(19.75)$ & $51.93(16.73)$ & $49.61(23.28)$ & $52.39(16.43)$ \\
& Word stem & $45.97(20.97)$ & $49.35(14.84)$ & $43.98(14.72)$ & $48.44(15.41)$ \\
& The & $2.77(1.63)$ & $2.92(1.49)$ & $3.14(2.17)$ & $3.19(2.07)$ \\
$\begin{array}{l}\text { Initial fixation letter } \\
\text { Saccade Amplitude }\end{array}$ & Word stem & $2.28(1.44)$ & $1.87(1.28)$ & $2.96(2.06)$ & $2.89(1.89)$ \\
$\begin{array}{l}\text { Initial fixation } \\
\text { location as \% of IA } \\
\text { extent }\end{array}$ & The & $50.26(26.75)$ & $49.23(23.93)$ & $50.01(26.75)$ & $46.31(25.18)$
\end{tabular}




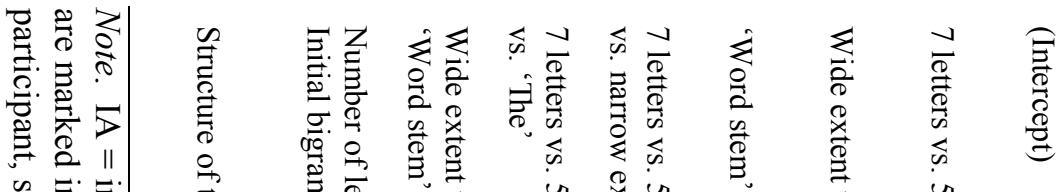

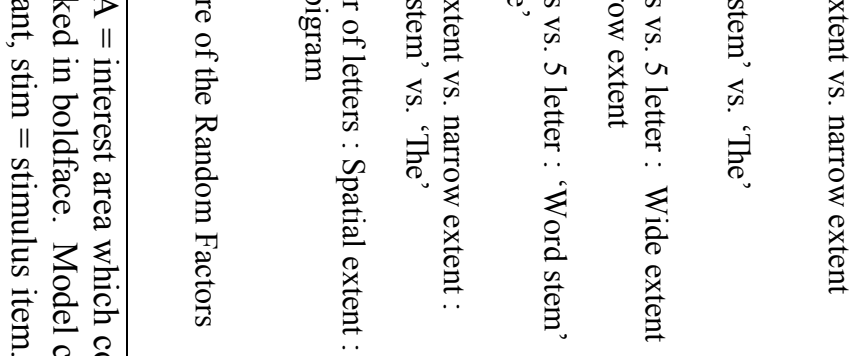

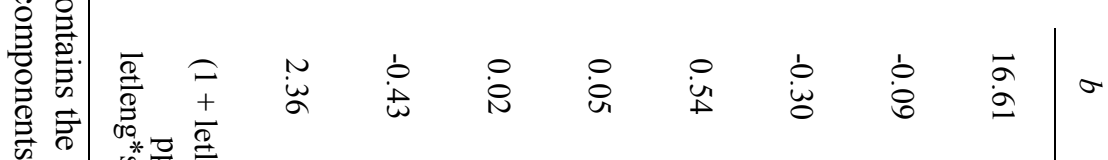

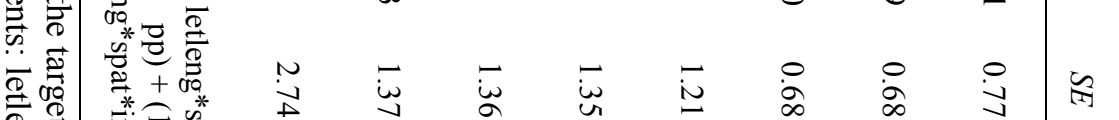

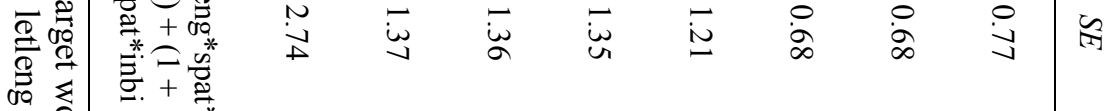

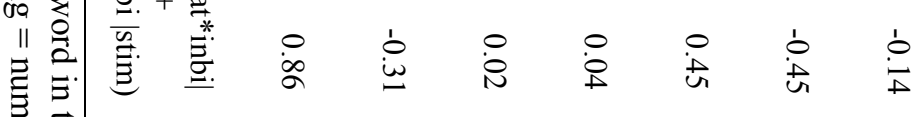

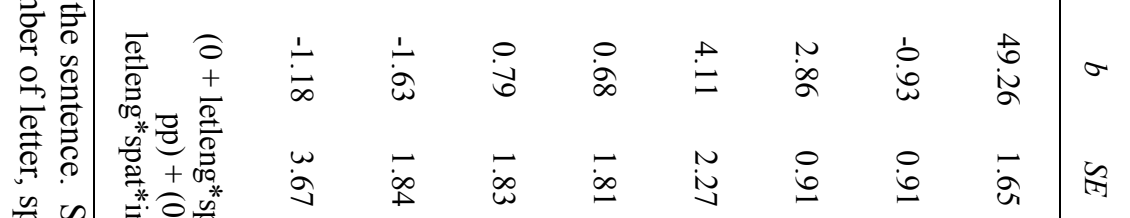

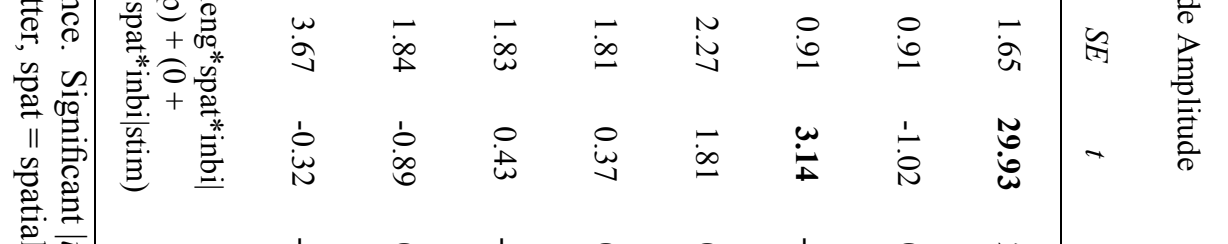

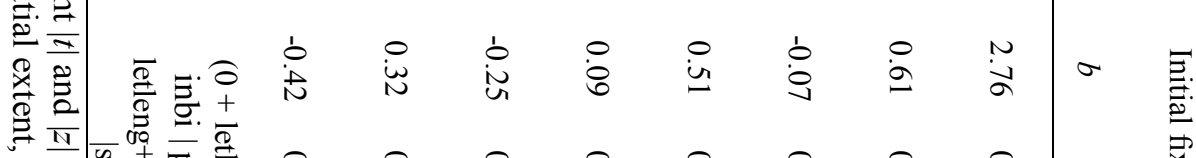

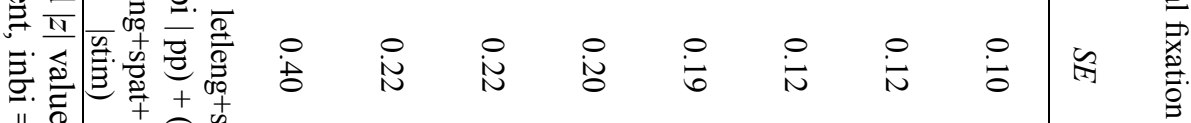

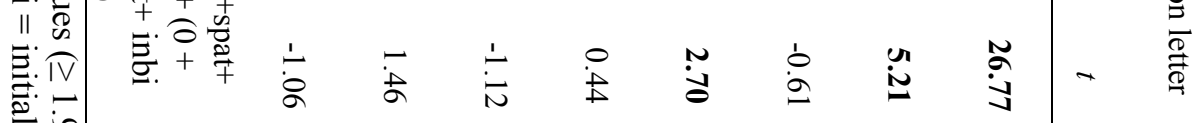

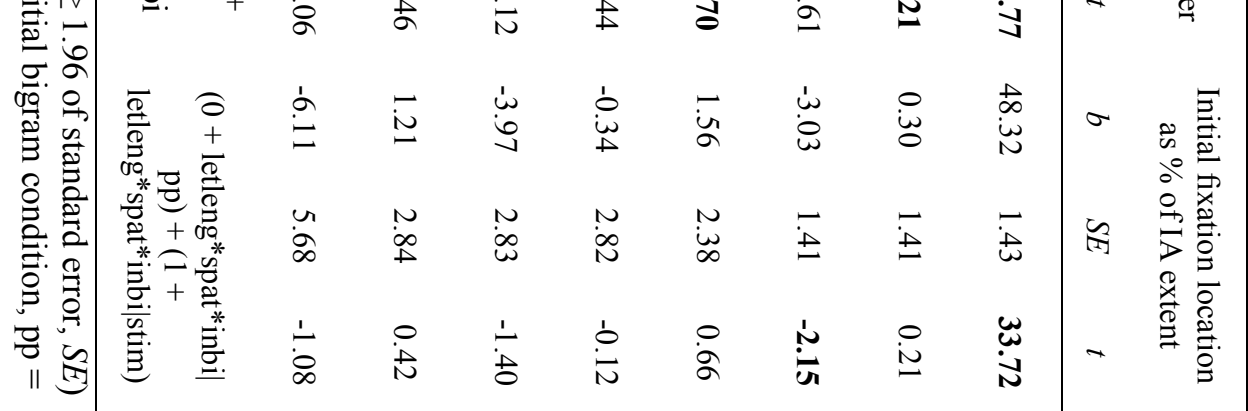


Figure 1

(A)

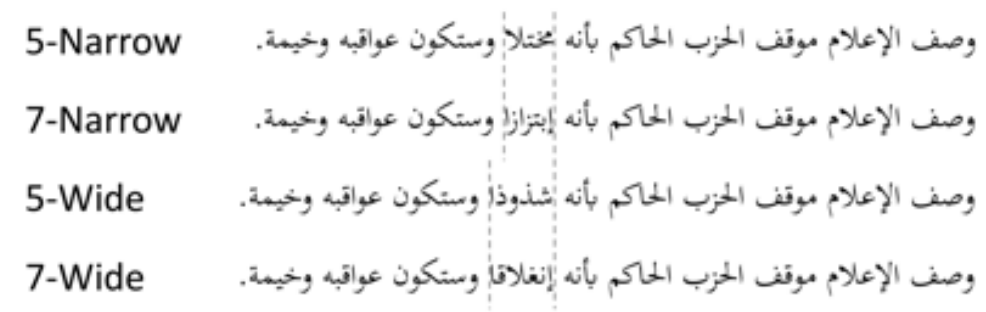

Translation: The media described the stance of the ruling party as imbalance / blackmail / deviant / [being] closed and its consequences will be dire.

(B)

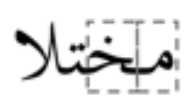

Naskh Script

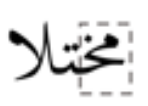

Ruq'a Script

(C)

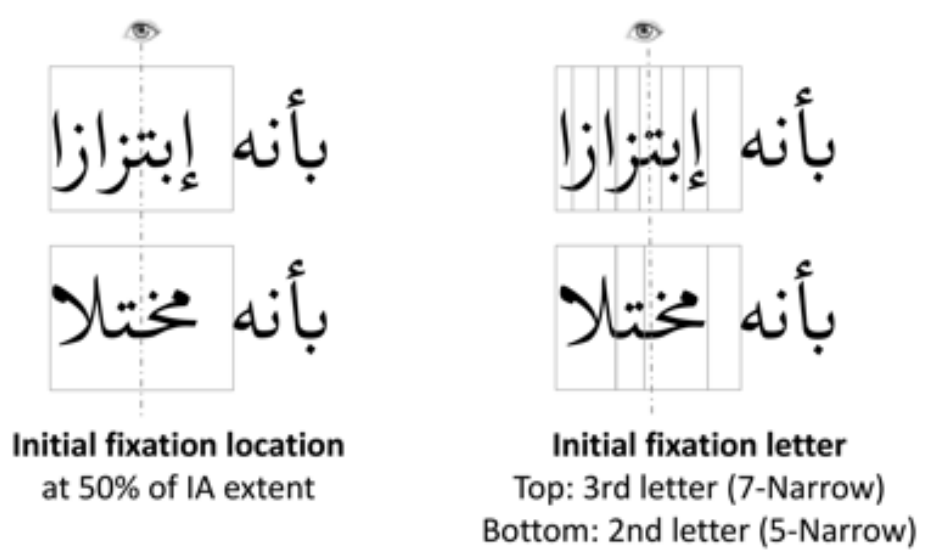

Figure 1. Panel (A) shows sample stimuli set and the translation. The dashed lines show the identical spatial extent of the 5- and 7-Narrow target words, and also the identical spatial extent of the wide conditions. Panel (B) shows sample of how letters are rendered to occupy the same vertical space in Ruq'a script, compared to Naskh script where the same two letters occupy different spaces. Panel (C) illustrates the letter-based, and the spatial measures (percentage) of initial fixation location. 
Figure 2

(A)

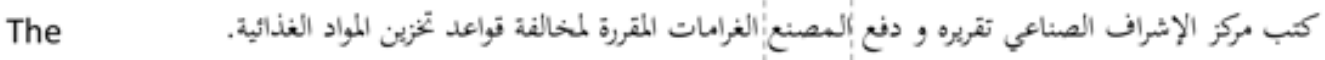

$$
\begin{aligned}
& \text { كتب مركز الاشراف الصناعي تقريره و دف اللمصنع:التعويضات المقرة بسبب الأخطاء التي تضمنها التقرير. To/for the } \\
& \text { كتب مركز الإشراف الصناعي تقريره و دف مصنعان: الغرامات المقروة لمخالفة قواعد تخزين المواد الغذائية. Word stem }
\end{aligned}
$$

\section{Translation:}

The industrial regulation center wrote its report and the factory paid the stated fines for violating the rules for food storage.

The industrial regulation center wrote its report and paid to the factory paid the stated fines because of the errors the report contained.

The industrial regulation center wrote its report and the factory paid the stated fines for violating the rules for food storage.

(B)

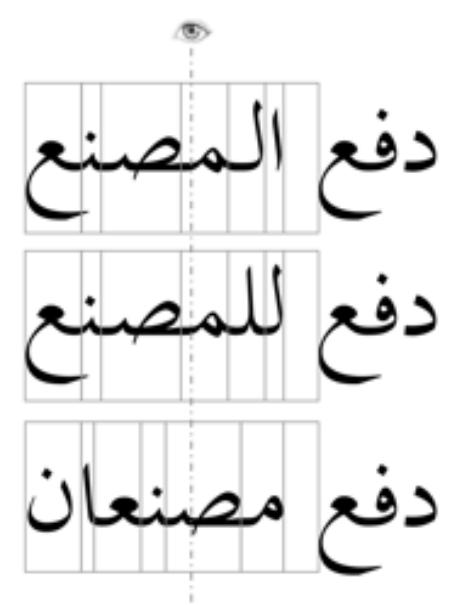

Figure 2. Panel (A) shows sample stimuli set and the translation. The dashed lines show the identical spatial extent of the target words that were also matched on the number of letters. Panel (B) illustrates how initial fixation location (the dashed line) when superimposed on letters reveals a difference in initial fixation letter, whereby fixations on the 3rd letter in ال the and لل to/for the conditions (top 2 conditions) corresponds to a fixation on the 2 nd letter in the word stem condition (bottom condition). 
Figure 3

\section{Initial Fixation Letter}

\section{Letters}

7 Letters
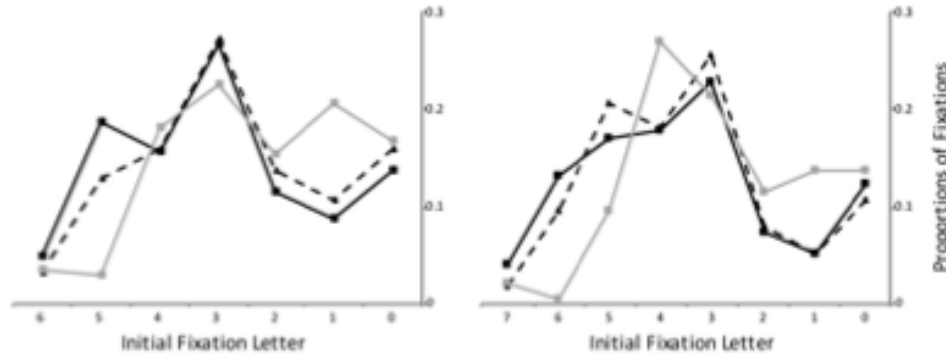

$\rightarrow$ For/To The

- Word Stem

Figure 3. The distributions of initial fixations on the letter, and the space before the word (marked as 0 on $\mathrm{x}$-axis) in Experiment 2. 
Figure 4

\section{Initial Fixation Location}

6 Letters

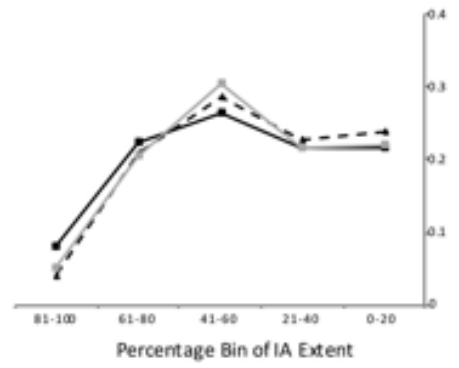

7 Letters

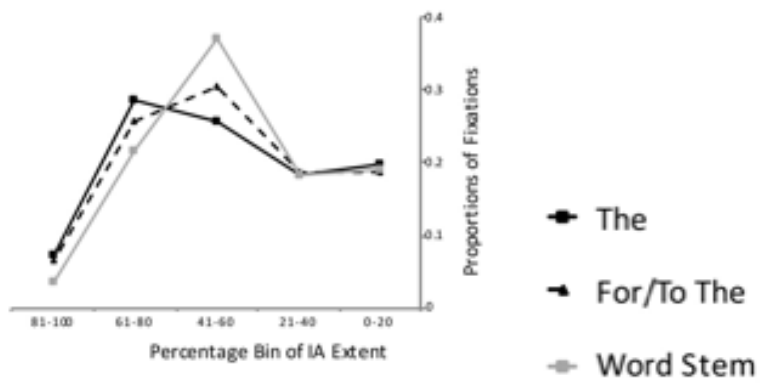

Figure 4. The distributions of initial fixations in $20 \%$ bins of the extent of the interest area containing the target word, including the space before the word in Experiment 2. 
Figure 5
Proportional Fonts
Arial
إختار الطفل اللون الأخضر لتلوين أرض الحديقة.
Times New Roman

$$
\text { إختار الطفل اللون الأخضر لتلوين أرض الحديقة. }
$$
Traditional Arabic

$$
\text { إختار الطفل اللون الأخضر لثلوين أرض الحديقة. }
$$
Lateef
إختار الطفل اللون الأخضر لتلوين أرض الحديقة.
Scheherazade
إختار الطفل اللون الأخضر لثلوين أرض الحديقة.

\section{Monospaced Fonts}

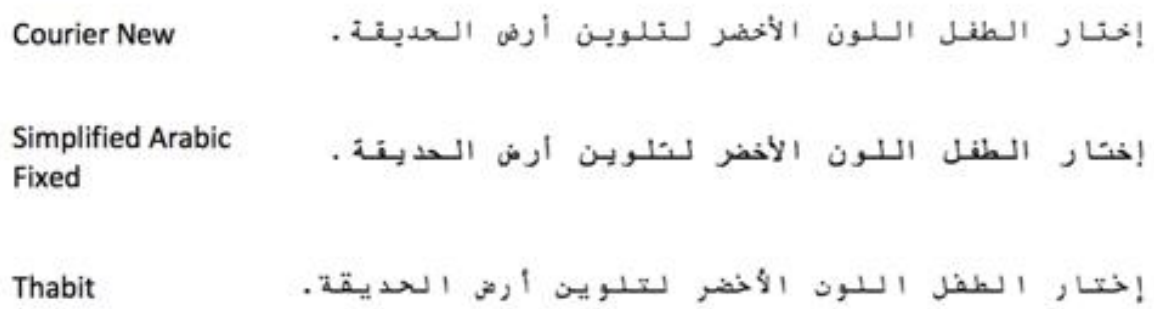

Translation: The child chose the green color to color the ground of the garden.

Figure 5. Sample sentence rendered in the proportional and monospaced fonts in the norming procedure. 


\section{Figure 6}

\section{Initial Fixation Letter}

\section{Letters}

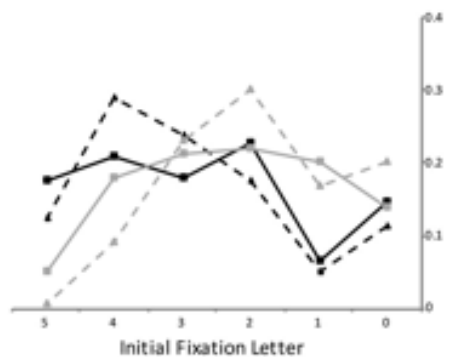

7 Letters

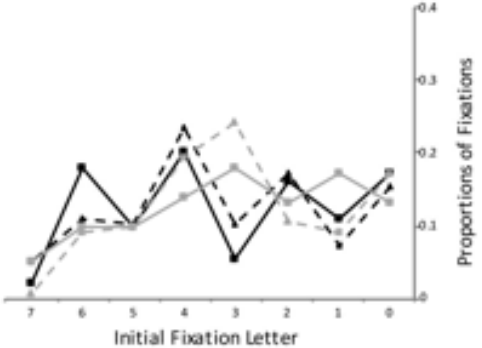

- The - Narrow

* The - Wide

- Word Stem - Narrow

- Word Stem - Wide

Figure 6. The distributions of initial fixations on the letter, and the space before the word (marked as 0 on x-axis) in Experiment 1 where stimuli set were split into 2 groups: beginning with ال the, or beginning with the word stem initial bigram. 


\section{Figure 7}

\section{Initial Fixation Location}

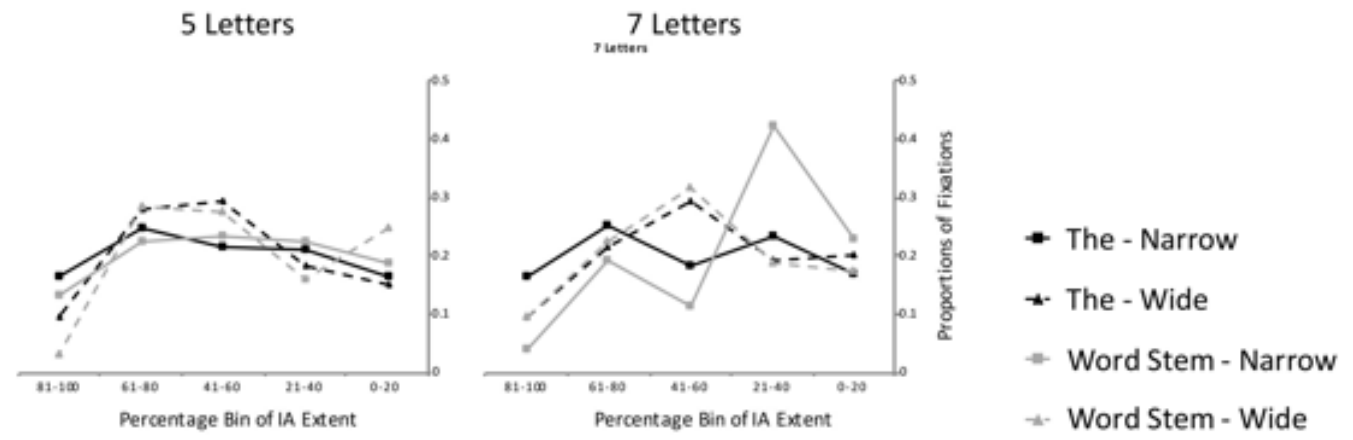

Figure 7. The distributions of initial fixations in $20 \%$ bins of the extent of the interest area containing the target word, including the space before the word in Experiment 1 where stimuli set were split into 2 groups: beginning with ال the, or beginning with the word stem initial bigram. 